\title{
Leaf classes, foliar phenology and life forms of selected woody species from the tropical forests of central and southern Eastern Ghats, India
}

\author{
Azariah Stephen ${ }^{1,2^{*}}$, Krishnamurthy Anupama ${ }^{1}$, Soupramanien Aravajy ${ }^{1}$ and Chrispus Livingstone ${ }^{2}$ \\ 1 Department of Ecology, French Institute of Pondicherry, 11, Saint Louis street, PB 33, Puducherry 605001 , India. \\ * Corresponding author's E-mail: stephen.a@ifpindia.org
}

\begin{abstract}
A checklist of selected woody species of Angiosperms is provided with the aim of classifying their life forms, foliar phenology and leaf classes from the tropical forests of central and southern Eastern Ghats, India. Though there are checklists available for the plants of the Eastern Ghats, a comprehensive listing of quantitative foliar measurements as done in other parts of the world, leading to valuable inputs for Plant Functional Type (PFT) classification, has not thus far been done for this key biogeographic zone of India. The list, gathered from 388 individual plants through the study area, encompasses 156 species and 3 infraspecific taxa which belong to 116 genera and 50 families. Of the total 159 taxa, 83 are evergreen and 76 are deciduous. 135 taxa are trees, 13 are shrubs 10 are climbing shrubs and one hemiparasite. Among the leaf classes of species, mesophyll dominated with 87 species, followed by notophyll (39), microphyll (24) and macrophyll (9). Hence, quantitative leaf trait measurements for selected woody species and the methodology for such studies in the tropics is the unique contribution of the present paper to the existing state-of-the-art.
\end{abstract}

\section{INTRODUCTION}

Understanding vegetation through plant functional types (PFTs) isimportantatatimeofincreased international concern on deforestation and fragmentation of forests strongly influenced by human impact. Leaves play a major role in the functional classification of plants because important functions such as photosynthesis, respiration and transpiration that greatly modify the morphology of the leaves and, in turn, the plant, occur here. Leaves closely interact with external environment and can be grouped into PFTs (Wright et al. 2004). As demonstrated clearly by Wright et al. (2004; 2005) and Barboni et al. (2004), actual trait measurements and analyses do add a distinct dimension to the field of functional ecology, especially from the point of view of conservation and management of forests and/or natural and human modified ecosystems (Lavorel et al. 1999; Landsberg et al. 1999). Functional grouping of species is also relevant for vegetation dynamics models that use leaf trait measurements for modeling global climate change and subsequent shifts in vegetation boundaries due to shifts of land use and climate change (Reich et al. 1997; Moorcroft et al. 2001; Bonan et al. 2002; Wright et al. 2004).

Though the Indian subcontinent, with its rich biodiversity, is one of the 12 mega-diversity regions in the world (McNeely et al. 1990), the high rate of degradation and change of forest cover, due to the so called developmental activities and the ever increasing human population in the forested areas (Jayakumar et al. 2002) results in increasing species loss that could lead to ecosystem collapse (Naeem 2002; Wackernagel et al. 2002). These changes have a number of effects on ecosystem processes (Naeem et al. 1999) and local populations in this region (Rao and Pullaiah 2007). Anthropogenic pressure in the form of clearing forest for mining, constructing tourist resorts and estate buildings, felling of selected trees for firewood, household furniture, fencing and for making minor agricultural implements (Kadavul and Parthasarathy 1999 a, b) leads to forest degradation and deforestation (Balaguru et al. 2006). It has been established by different studies (Hooper and Vitousek 1997, 1998; Tilman et al. 1997; Hooper et al. 2005; McLaren 2006) that the functional roles of plant species have to be first understood, to establish their ecological niche in the complex process of ecosystem dynamics, to arrive at any means of forest conservation. Though there are some checklists available for the plant species of the Eastern Ghats (Muthumperumal and Parthasarathy 2009; Pragasan and Parthasarathy 2009; Reddy et al. 2009), the unique contribution of the present paper to the existing state-of-the-art is to present, for a selection of these species, comprehensive quantitative foliar measurements as done in other parts of the world (Roderick et al. 1999; Wright et al. 2004) leading to valuable inputs towards an eventually more rigorous PFT classification, for this key biogeographic zone of India; a definitive protocol for such measurements, rare to non existent in the Indian context is an important methodological by-product.

\section{MATERIALS AND Methods \\ Study area}

The Eastern Ghats $\left(10^{\circ}\right.$ to $22^{\circ} \mathrm{N} ; 76^{\circ} 50^{\prime}$ to $\left.86^{\circ} 30^{\prime} \mathrm{E}\right)$ is a discontinuous range of mountains along the eastern coast of Peninsular India extending through the states of Orissa (25\% of the range area), Andhra Pradesh (48\%), Karnataka (2\%) and Tamil Nadu (24\%) (Figure 1). It falls in one of the 10 biogeographic zones of India (Rodgers and Panwar 1988) and also in one of the 8 phytogeographical regions of India (Chatterjee 1939) renamed as eco-floristic zones (Blasco 1979). The Eastern Ghats including lowland 
areas encompasses an area of $c a .260,000 \mathrm{~km}^{2}$. Its area of extent is 1,130 km North-South and 1,050 km East-West. Based on geological and tectonic considerations, this mountain range is formed of four sections or provinces that are not fully or clearly established (Krishnan 1961; 1974; Meher-Homji 2001; Dobmeier and Raith 2003; Mukhopadhyay and Basak 2009). The Eastern Ghats, falling under tropical monsoon climate, receives rainfall from both south-west monsoon and north-east retreating monsoon. The natural vegetation, though primarily dominated by tropical deciduous species, may be grouped into Evergreen forests, Tropical semi-evergreen forests, Tropical moist deciduous forests, Dry deciduous forests, Tropical dry evergreen forests and Dry evergreen scrubs (Champion and Seth 1968; Legris and Meher-Homji 1984; Pragasan and Parthasarathy 2009).

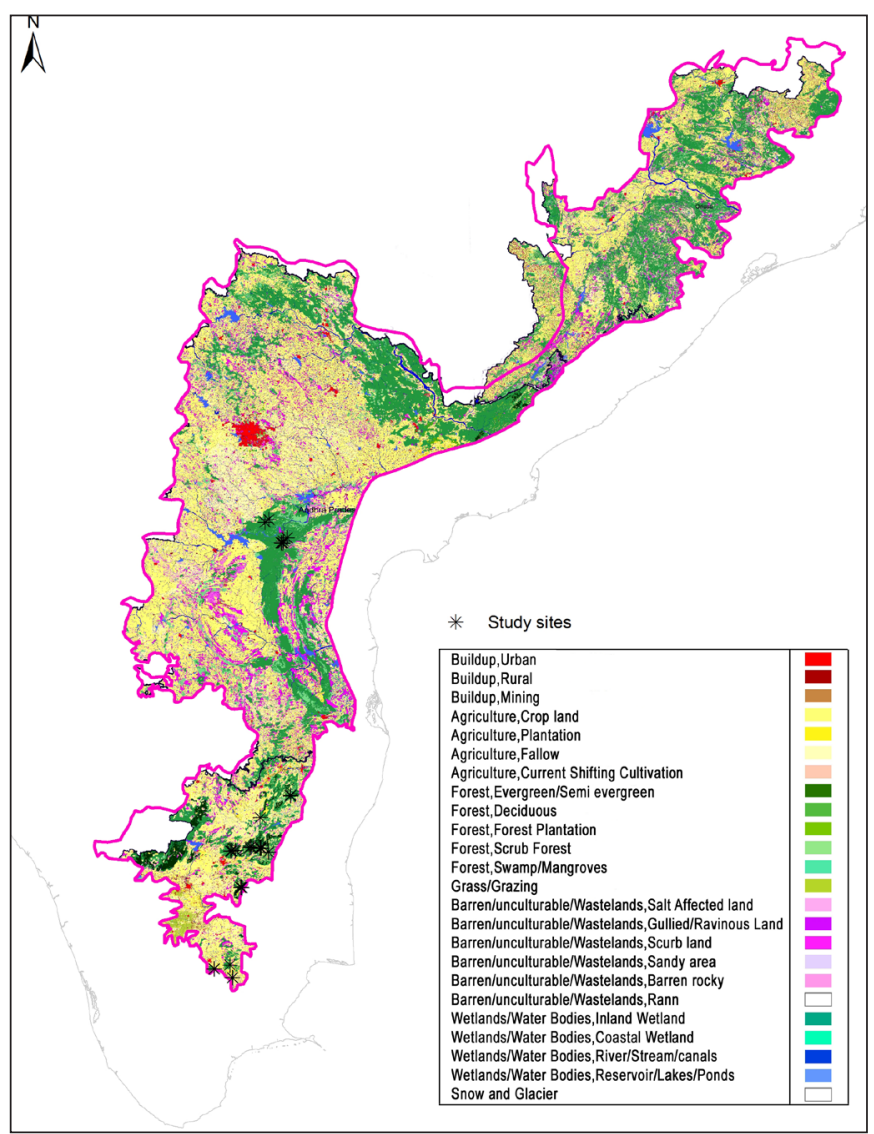

FIGURE 1. Map showing the study sites of central and southern Eastern Ghats (modified after NRSC (2006)).
The present study focuses on the area consisting of the Nallamalai hills (Andhra Pradesh) section of central Eastern Ghats and Javadi, Kalrayan, Chitteri, Shevaroy, Kolli and Pachamalai hills (Tamil Nadu) section of southern Eastern Ghats (Figure 1). The study sites were selected based on the floristic composition and priority of conservation (Kadavul and Parthasarathy. 1999a, b; Jayakumar et al. 2002; Natarajan et al. 2004; Soosairaj et al. 2004, 2007; Reddy et al. 2008; Pragasan and Parthasarathy 2009). Out of 38 sites, 30 sites are from the disjunct hillocks of the southern Eastern Ghats and 8 sites from the more or less contiguous Nallamalai hills of central Eastern Ghats representative of the major vegetation types of the region (Reddy et al. 2008). Although the altitude of these studied sites ranges from 16 to $1362 \mathrm{~m}$ a.s.l., the variations in the mean annual rainfall are within the narrow range of 800 to $1100 \mathrm{~mm}$ only.

\section{Data Collection}

Leaf trait measurements were carried out and phenology observed on a set of plant species selected based on the following criteria: 1) woody species, mainly trees and also some important shrubs and lianas were selected; 2) simple leaves were selected preferentially not only for ease of measurements but also because the existing protocols for trait measurements recommended this (Turner and Tan, 1991; Roderick et al. 1999; Cornelissen et al. 2003); 3) some species with large compound leaves were also chosen, as species with compound leaves were dominant in the study area (Reddy et al. 2009) - further because it is important to standardize the protocol as well. Phenology observations were cross-checked, where required, with record of individuals in the herbarium of French Institute of Pondicherry (HIFP).

Standard protocols proposed by Cornelissen et al. (2003); Garnier et al. (2001) and Roderick et al. (1999) have been adopted with minor changes for leaf trait measurements. Leaf-size was determined using the geometric leaf-size classification of Raunkiaer (1934) as modified by Webb (1959) and recently by Gillison (2002). Six leaf classes were recognized by Raunkiaer (1934). This was later modified into seven by the addition of one more leaf size class 'notophyll' by Webb (1959). Recently Gillison (2002) recognized 10 leaf size classes (Table 1). Floras such as Hooker (1872-1879), Gamble and Fischer (1915-1935), Nair and Henry (1983), Henry et al. (1987)

TABLE 1. Derivation and correspondence of leaf categories used in study and the leaf classes found in the study area highlighted.

\begin{tabular}{lllll}
\hline CATEGORY & DIMENSIONS (MM $\left.{ }^{2}\right)$ & RAUNKIAER (1934) & WEBB (1959) & GILLISON (2002) \\
\hline 1 & & & & No repeating leaf units \\
2 & $<2$ & & & Picophyll \\
3 & $2-25$ & Nanophyll & Leptophyll \\
4 & $25-225$ & Microphyll & Nanophyll & Nanophyll \\
5 & $225-2025$ & & Microphyll & Microphyll \\
6 & $2025-4500$ & & Notophyll & Notophyll \\
7 & $4500-18,200$ & & Mesophyll & Mesophyll \\
8 & $18,200-36,400$ & Macrophyll & Platyphyll \\
9 & $36,400-18 \times 10^{4}$ & & & Macrophyll \\
10 & $>18 \times 10^{4}$ & Megaphyll & & Megaphyll \\
\hline
\end{tabular}


and Matthew (1983) were used for identification of plant species. Voucher specimens were collected and confirmed with the HIFP and deposited there. We have used APG III (2009) classification for enumeration of families. Leaf size, thickness and weight were measured on the field using hand drawn sketches, thickness gauge and portable weighing machine respectively. The hand drawn sketches were brought to the lab and the area was measured using foliar surface area calculation software developed by French Institute of Pondicherry. Leaf area alone is considered for the leaf size classification in this study.

Totally, 143 species with simple leaves and 16 species with compound leaves were selected for the present study. For simple leaves, four mature "full sun" leaves (Cornelissen et al. 2003) were measured from every individual. Similarly for compound leaves, leaflet from the species was considered as the unit of study as it is analogous to the simple leaf (Raunkiaer 1934; Cain et al. 1956). The average value was taken as final value for the individual. Southern Eastern Ghats were sampled during February to April 2007 whereas Central Eastern Ghats were sampled in January 2008. All leaf samples were collected between 09:00 $\mathrm{h}$ and 14:00 $\mathrm{h}$ in order to avoid any effect of temperature, irradiance or vapour pressure deficit of the air on the leaf traits. For most of the species, photographic documentation was carried out and is presented (Figures 2-10), with an emphasis on the species not photo-documented previously. Pearson's correlation coefficient test (parametric test) was done to know the correlation between various parameters in XLStat ${ }^{\circledR}$. Official permissions to carry out the field works for this study were provided by the Headquarters and Divisional offices of the forest departments of Andhra Pradesh and Tamil Nadu.

\section{RESULTS AND DiscuSSION}

388 individuals from 38 sites of southern and central
Eastern Ghats were measured for leaf traits (Figure 1; Supporting Information). This represented 156 species and 3 infraspecific taxa which fall under 116 genera, 50 families and four leaf classes (Table 2).

Of the 159 woody species that were studied, 135 were trees, 13 were shrubs 10 were climbing shrubs and one hemiparasite (Table 2). Among the different life forms, $54 \%$ of the taxa measured were mesophyllous, while $25 \%$ were notophyllous, $15 \%$ microphyllous, and only $6 \%$ were macrophyllous (Table 2). The higher proportion of mesophylls and the moderate proportions of microphylls and notophylls are characteristic features of tropical regions (Cain et al. 1956; Cain and de Oliveira Castro 1959; Richards 1957; Webb 1959; Malhado et al. 2009) and this is true for our study area. In the list, leaves of two species of Bauhinia (B. racemosa and B. vahlii) are considered as simple as in Saldanha (1984) though most of the taxonomists term them compound.

Reddy et al. (2009) studied the tree wealth of Eastern Ghats of Andhra Pradesh and came up with an updated list of 510 tree species, of which 135 are with compound leaves. The present study includes 68 species from central Eastern Ghats of which 62 species are listed by Reddy et al. (2009) and of which 9 are with compound leaves; the remaining species are climbing shrubs (5) and shrub (1) (Table 2). Foliar phenology and leaf classes of 68 species from central Eastern Ghats are new additions to the existing list of species by Reddy et al. (2009). In our study 10 species of climbing shrubs are included. Out of these 10 species, 5 are from southern Eastern Ghats, 4 are from central Eastern Ghats, 1 species (Hiptage benghalensis) occurs both in southern as well as central Eastern Ghats. Pragasan and Parthasarathy (2009) reported 272 species from tropical forests of southern Eastern Ghats of Tamil Nadu. The present checklist covers only 134 species from southern Eastern Ghats; however 40 species were not listed in that earlier study (Table 2).

TABLE 2. Enumeration of life form, phenology, leaf form and leaf class of selected woody species from the hills of the southern and central Eastern Ghats, India. (T: tree; S: shrub; CS: climbing shrub; HP: hemiparasite; D: deciduous; E: evergreen; Si: Simple leaves; C: Compound leaves; Mi: microphyll; N: notophyll; Me: mesophyll; Ma: macrophyll; ${ }^{*}$ Herbier Institut Français de Pondichéry. Highlighted species are hitherto new report to Eastern Ghats).

\begin{tabular}{|c|c|c|c|c|c|c|}
\hline S. NO. & BOTANICAL NAME & $\begin{array}{c}\text { LIFE } \\
\text { FORM }\end{array}$ & PHENOLOGY & $\begin{array}{l}\text { LEAF } \\
\text { FORM }\end{array}$ & $\begin{array}{l}\text { LEAF } \\
\text { CLASS }\end{array}$ & * HIFP NO. \\
\hline \multicolumn{7}{|c|}{ Anacardiaceae } \\
\hline 1 & Buchanania axillaris (Desr.) Ramam. & $\mathrm{T}$ & $\mathrm{D}$ & $\mathrm{Si}$ & $\mathrm{N}$ & $24391,24404,25336$ \\
\hline 2 & Buchanania lanzan Spreng. & $\mathrm{T}$ & $\mathrm{E}$ & $\mathrm{Si}$ & $\mathrm{Me}$ & $24435,24499,24514,24525$ \\
\hline 3 & Mangifera indica $\mathrm{L}$. & $\mathrm{T}$ & $\mathrm{E}$ & $\mathrm{Si}$ & $\mathrm{Me}$ & $24480,25519,24534$ \\
\hline 4 & Nothopegia beddomei Gamble & $\mathrm{T}$ & $\mathrm{E}$ & $\mathrm{Si}$ & $\mathrm{N}$ & 24507,25458 \\
\hline 5 & Nothopegia heyneana (Hook. f.) Gamble & $\mathrm{T}$ & $\mathrm{E}$ & $\mathrm{Si}$ & Mi & 25814 \\
\hline 6 & Semecarpus anacardium L. f. & $\mathrm{T}$ & $\mathrm{D}$ & $\mathrm{Si}$ & Ma & 24423,24577 \\
\hline \multicolumn{7}{|l|}{ Annonaceae } \\
\hline 7 & Miliusa tomentosa (Roxb.) J. Sinclair & $\mathrm{T}$ & $\mathrm{E}$ & $\mathrm{Si}$ & $\mathrm{Mi}$ & 24413 \\
\hline 8 & Polyalthia cerasoides (Roxb.) Bedd. & $\mathrm{T}$ & $\mathrm{D}$ & $\mathrm{Si}$ & $\mathrm{Me}$ & $24483,25290,24561,24580$ \\
\hline 9 & Uvaria narum Wall. & $\mathrm{CS}$ & $\mathrm{E}$ & Si & $\mathrm{Me}$ & 25522 \\
\hline \multicolumn{7}{|l|}{ Apocynaceae } \\
\hline 10 & Holarrhena pubescens Wall. & $\mathrm{T}$ & $\mathrm{D}$ & $\mathrm{Si}$ & $\mathrm{Me}$ & 24569 \\
\hline 11 & Wrightia arborea (Dennst.) Mabb. & $\mathrm{T}$ & $\mathrm{D}$ & $\mathrm{Si}$ & $\mathrm{Me}$ & 25292 \\
\hline 12 & Wrightia tinctoria (Roxb.) R. Br. & $\mathrm{T}$ & $\mathrm{D}$ & $\mathrm{Si}$ & $\mathrm{Me}$ & 24442,24564 \\
\hline \multicolumn{7}{|l|}{ Araliaceae } \\
\hline 13 & Schefflera stellata (Gaertn.) Harms & $\mathrm{CS}$ & $\mathrm{E}$ & $\mathrm{C}$ & $\mathrm{Me}$ & 24430 \\
\hline \multicolumn{7}{|l|}{ Asteraceae } \\
\hline 14 & Vernonia arborea Buch.-Ham. & $\mathrm{T}$ & $\mathrm{D}$ & $\mathrm{Si}$ & Ma & 24471 \\
\hline
\end{tabular}


TABLE 2. CONTINUED.

\begin{tabular}{|c|c|c|c|c|c|c|}
\hline S. NO. & BOTANICAL NAME & $\begin{array}{l}\text { LIFE } \\
\text { FORM }\end{array}$ & PHENOLOGY & $\begin{array}{l}\text { LEAF } \\
\text { FORM }\end{array}$ & $\begin{array}{l}\text { LEAF } \\
\text { CLASS }\end{array}$ & * HIFP NO. \\
\hline \multicolumn{7}{|l|}{ Bignoniaceae } \\
\hline 15 & Stereospermum personatum (Hassk.) Chatterjee & $\mathrm{T}$ & $\mathrm{D}$ & $\mathrm{C}$ & $\mathrm{N}$ & 24521 \\
\hline \multicolumn{7}{|l|}{ Boraginaceae } \\
\hline 16 & Cordia dichotoma Forst. & $\mathrm{T}$ & $\mathrm{D}$ & $\mathrm{Si}$ & $\mathrm{Me}$ & $24539,24552,25308,24573$ \\
\hline 17 & Cordia macleodii Hook. f et Thoms. & $\mathrm{T}$ & $\mathrm{D}$ & $\mathrm{Si}$ & $\mathrm{Me}$ & 24398 \\
\hline 18 & Cordia wallichii G. Don & $\mathrm{T}$ & $\mathrm{D}$ & $\mathrm{Si}$ & Ma & 25318 \\
\hline 19 & Ehretia canarensis (Clarke) Gamble & $\mathrm{T}$ & $\mathrm{D}$ & $\mathrm{Si}$ & $\mathrm{Me}$ & 24545,24562 \\
\hline 20 & Ehretia laevis Roxb. & $\mathrm{T}$ & $\mathrm{D}$ & $\mathrm{Si}$ & $\mathrm{N}$ & 24392 \\
\hline 21 & Ehretia ovalifolia Hassk. & $\mathrm{T}$ & $\mathrm{D}$ & $\mathrm{Si}$ & $\mathrm{N}$ & 25807 \\
\hline \multicolumn{7}{|l|}{ Burseraceae } \\
\hline 22 & Commiphora berryi (Arn.) Engler & $\mathrm{T}$ & $\mathrm{D}$ & $\mathrm{C}$ & $\mathrm{N}$ & 24563 \\
\hline \multicolumn{7}{|l|}{ Buxaceae } \\
\hline 23 & Sarcococca trinervia Wight & $\mathrm{S}$ & $\mathrm{E}$ & $\mathrm{Si}$ & $\mathrm{Me}$ & 24492 \\
\hline \multicolumn{7}{|c|}{ Caprifoliaceae } \\
\hline 24 & Viburnum punctatum Ham. ex G. Don & $\mathrm{T}$ & $\mathrm{E}$ & $\mathrm{S}$ & $\mathrm{Me}$ & 24493 \\
\hline \multicolumn{7}{|l|}{ Celastraceae } \\
\hline 25 & Cassine glauca (Rottb.) Kuntze & $\mathrm{T}$ & $\mathrm{E}$ & $\mathrm{Si}$ & $\mathrm{Me}$ & 24400,25311 \\
\hline 26 & Celastrus paniculatus Willd. & $\mathrm{CS}$ & $\mathrm{D}$ & $\mathrm{Si}$ & $\mathrm{N}$ & 24567 \\
\hline 27 & Maytenus emarginata (Willd.) Ding Hou & $\mathrm{S}$ & $\mathrm{D}$ & $\mathrm{Si}$ & $\mathrm{N}$ & $24421,24512,25512,25730$ \\
\hline \multicolumn{7}{|l|}{ Clusiaceae } \\
\hline 28 & Garcinia spicata (Wight and Arn.) Hook. f. & $\mathrm{T}$ & $\mathrm{E}$ & $\mathrm{Si}$ & $\mathrm{Me}$ & 24370 \\
\hline \multicolumn{7}{|c|}{ Combretaceae } \\
\hline 29 & $\begin{array}{l}\text { Anogeissus latifolia (Roxb. ex DC.) Wall. ex Guill. } \\
\text { and Perr. }\end{array}$ & $\mathrm{T}$ & $\mathrm{D}$ & $\mathrm{Si}$ & $\mathrm{Me}$ & $24382,24395,24427,25734,24543$ \\
\hline 30 & Terminalia alata Heyne ex Roth. & $\mathrm{T}$ & $\mathrm{D}$ & $\mathrm{Si}$ & $\mathrm{Me}$ & $24393,24424,24574$ \\
\hline 31 & $\begin{array}{l}\text { Terminalia arjuna (Roxb. ex DC.) Wight and } \\
\text { Arn. }\end{array}$ & $\mathrm{T}$ & $\mathrm{D}$ & $\mathrm{Si}$ & $\mathrm{Me}$ & $25321,25517,24530$ \\
\hline 32 & Terminalia chebula Retz. & $\mathrm{T}$ & $\mathrm{D}$ & $\mathrm{Si}$ & $\mathrm{Me}$ & 24425 \\
\hline 33 & Terminalia paniculata Roth. & $\mathrm{T}$ & $\mathrm{D}$ & $\mathrm{Si}$ & $\mathrm{Me}$ & 25304 \\
\hline \multicolumn{7}{|l|}{ Cornaceae } \\
\hline 34 & Alangium salvifolium (L. f.) Wang. & $\mathrm{T}$ & $\mathrm{D}$ & $\mathrm{Si}$ & $\mathrm{N}$ & 24565 \\
\hline \multicolumn{7}{|c|}{ Daphniphyllaceae } \\
\hline 35 & Daphniphyllum neilgherrense (Wt.) Rosenth & $\mathrm{T}$ & $\mathrm{E}$ & $\mathrm{Si}$ & Me & 24490 \\
\hline \multicolumn{7}{|c|}{ Dipterocarpaceae } \\
\hline 36 & Shorea tumbaggaia Roxb. & $\mathrm{T}$ & $\mathrm{E}$ & $\mathrm{Si}$ & $\mathrm{Me}$ & 24418 \\
\hline \multicolumn{7}{|l|}{ Ebenaceae } \\
\hline 37 & Diospyros angustifolia (Miq.) Kosterm & $\mathrm{T}$ & $\mathrm{E}$ & $\mathrm{Si}$ & $\mathrm{Mi}$ & 24466 \\
\hline 38 & Diospyros chloroxylon Roxb. & $\mathrm{T}$ & $\mathrm{E}$ & $\mathrm{Si}$ & $\mathrm{Me}$ & 24554,25302 \\
\hline 39 & Diospyros ebenum J. Koenig ex Retz. & $\mathrm{T}$ & $\mathrm{E}$ & $\mathrm{Si}$ & $\mathrm{N}$ & 25815 \\
\hline 40 & Diospyros melanoxylon Roxb. & $\mathrm{T}$ & $\mathrm{D}$ & $\mathrm{Si}$ & $\mathrm{Me}$ & $24397,25509,25315$ \\
\hline 41 & Diospyros montana Roxb. & $\mathrm{T}$ & $\mathrm{D}$ & $\mathrm{Si}$ & $\mathrm{Mi}$ & 24403,24449 \\
\hline 42 & Diospyros ovalifolia Wight & $\mathrm{T}$ & $\mathrm{E}$ & $\mathrm{Si}$ & $\mathrm{N}$ & 24463,24476 \\
\hline 43 & Diospyros peregrina (Gaertn.) Gurke & $\mathrm{T}$ & $\mathrm{E}$ & $\mathrm{Si}$ & $\mathrm{Me}$ & 25819 \\
\hline \multicolumn{7}{|c|}{ Elaeocarpaceae } \\
\hline 44 & Elaeocarpus serratus L. & $\mathrm{T}$ & $\mathrm{E}$ & $\mathrm{Si}$ & $\mathrm{Me}$ & $25310,24494,24495$ \\
\hline \multicolumn{7}{|l|}{ Ericaceae } \\
\hline 45 & Vaccinium neilgherrense $\mathrm{Wt}$. & $\mathrm{S}$ & $\mathrm{E}$ & $\mathrm{Si}$ & Mi & 24486 \\
\hline \multicolumn{7}{|c|}{ Erythroxylaceae } \\
\hline 46 & Erythroxylum monogynum Roxb. & $\mathrm{T}$ & $\mathrm{D}$ & $\mathrm{Si}$ & Mi & $\begin{array}{l}24409,24405,24450,24518,25316, \\
24584\end{array}$ \\
\hline \multicolumn{7}{|c|}{ Euphorbiaceae } \\
\hline 47 & Antidesma montanum Blume & $\mathrm{T}$ & $\mathrm{E}$ & $\mathrm{Si}$ & $\mathrm{Me}$ & 24497 \\
\hline 48 & Bischofia javanica Blume & $\mathrm{T}$ & $\mathrm{E}$ & $\mathrm{C}$ & $\mathrm{Me}$ & 25289 \\
\hline 49 & Bridelia retusa (L.) Spreng. & $\mathrm{T}$ & $\mathrm{D}$ & $\mathrm{Si}$ & $\mathrm{Me}$ & 24550,24551 \\
\hline 50 & Croton laccifer L. & $\mathrm{T}$ & $\mathrm{D}$ & $\mathrm{Si}$ & $\mathrm{Me}$ & 24467 \\
\hline 51 & $\begin{array}{l}\text { Drypetes sepiaria (Wight and Arn.) Pax and K. } \\
\text { Hoffm. }\end{array}$ & $\mathrm{T}$ & $\mathrm{E}$ & $\mathrm{Si}$ & $\mathrm{Mi}$ & $24380,24388,24445,25456,25731$ \\
\hline 52 & Euphorbia nivulia Buch-Ham & $\mathrm{T}$ & $\mathrm{D}$ & $\mathrm{Si}$ & $\mathrm{N}$ & 24369 \\
\hline 53 & Flueggea leucopyrus Willd. & $\mathrm{S}$ & $\mathrm{D}$ & $\mathrm{Si}$ & $\mathrm{Mi}$ & 24406 \\
\hline 54 & Glochidion ellipticum Wight & $\mathrm{T}$ & $\mathrm{E}$ & $\mathrm{Si}$ & $\mathrm{Me}$ & 25326 \\
\hline 55 & Macaranga peltata (Roxb.) Muell.-Arg. & $\mathrm{T}$ & $\mathrm{E}$ & $\mathrm{Si}$ & $\mathrm{Ma}$ & 25735 \\
\hline
\end{tabular}


TABle 2. Continued.

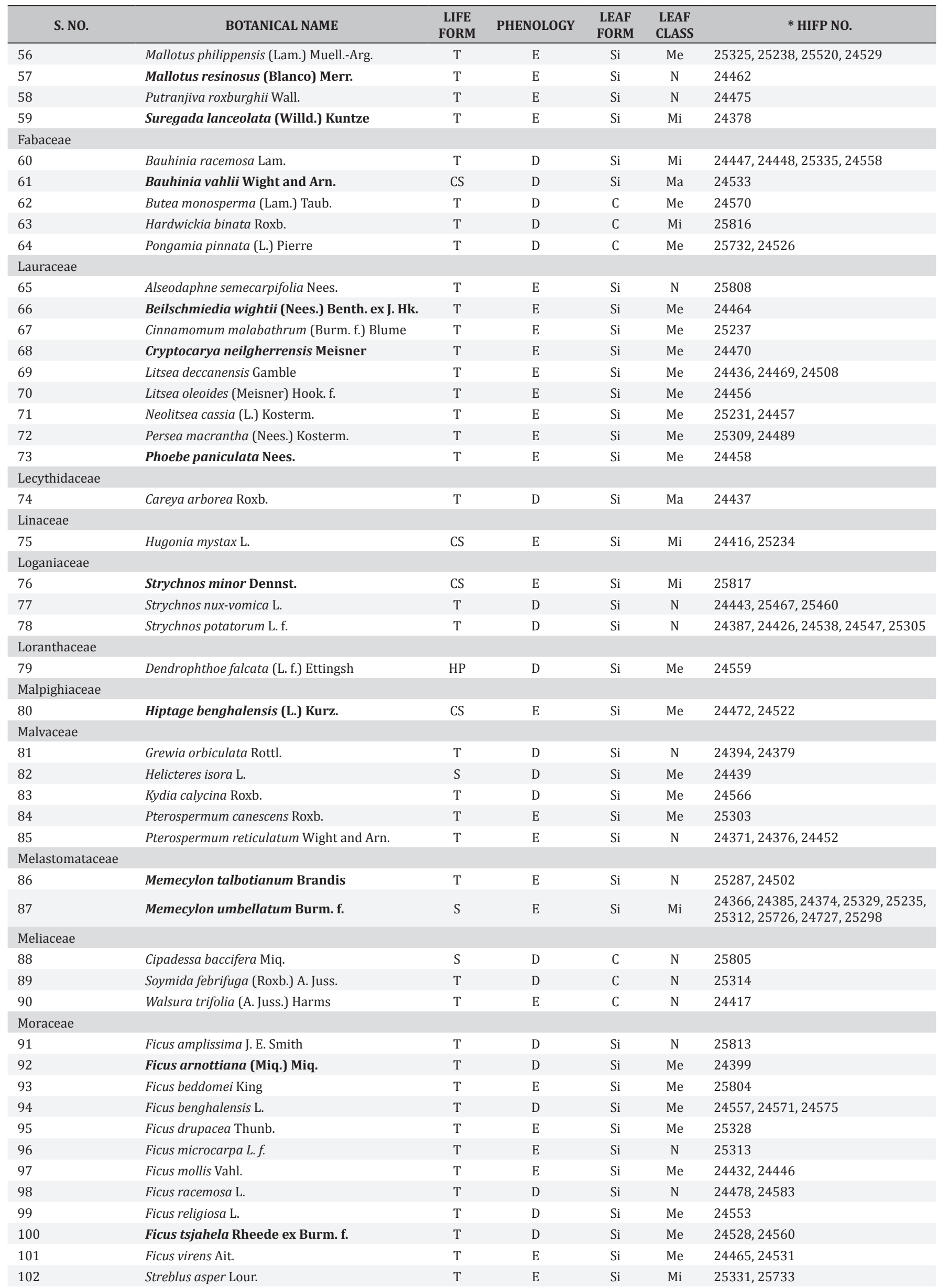


TABle 2. Continued.

\begin{tabular}{|c|c|c|c|c|c|c|}
\hline S. NO. & BOTANICAL NAME & $\begin{array}{c}\text { LIFE } \\
\text { FORM } \\
\end{array}$ & PHENOLOGY & $\begin{array}{l}\text { LEAF } \\
\text { FORM } \\
\end{array}$ & $\begin{array}{l}\text { LEAF } \\
\text { CLASS }\end{array}$ & * HIFP NO. \\
\hline \multicolumn{7}{|l|}{ Myristicaceae } \\
\hline 103 & Myristica dactyloides Gaertn. & $\mathrm{T}$ & $\mathrm{E}$ & $\mathrm{Si}$ & $\mathrm{Me}$ & 25330 \\
\hline \multicolumn{7}{|l|}{ Myrtaceae } \\
\hline 104 & Syzygium alternifolium (Wight) Walp. & $\mathrm{T}$ & $\mathrm{D}$ & $\mathrm{Si}$ & $\mathrm{Me}$ & 24419 \\
\hline 105 & Syzygium cumini (L.) Skeels & $\mathrm{T}$ & $\mathrm{E}$ & $\mathrm{Si}$ & $\mathrm{N}$ & $\begin{array}{l}24372,25322,24496,24517,25518 \\
24527,24556,25307,24585\end{array}$ \\
\hline \multicolumn{7}{|l|}{ Ochnaceae } \\
\hline 106 & Ochna obtusata DC. & $\mathrm{T}$ & $\mathrm{D}$ & $\mathrm{Si}$ & $\mathrm{Me}$ & 24401 \\
\hline \multicolumn{7}{|l|}{ Olacaceae } \\
\hline 107 & Olax scandens Roxb. & $\mathrm{CS}$ & $\mathrm{D}$ & $\mathrm{Si}$ & $\mathrm{Mi}$ & 25299 \\
\hline \multicolumn{7}{|l|}{ Oleaceae } \\
\hline 108 & Chionanthus mala-elengi (Dennst.) P. S. Green & $\mathrm{T}$ & $\mathrm{E}$ & $\mathrm{Si}$ & $\mathrm{N}$ & 25812 \\
\hline 109 & Chionanthus ramiflora Roxb. & $\mathrm{T}$ & $\mathrm{E}$ & $\mathrm{Si}$ & $\mathrm{Me}$ & $24414,24461,25332,24513$ \\
\hline 110 & Chionanthus zeylanica $\mathrm{L}$. & $\mathrm{T}$ & $\mathrm{E}$ & $\mathrm{Si}$ & $\mathrm{N}$ & 24367,24407 \\
\hline 111 & Ligustrum perrottetii DC. & $\mathrm{S}$ & $\mathrm{E}$ & $\mathrm{Si}$ & $\mathrm{Mi}$ & $25323,24487,25333$ \\
\hline 112 & Olea dioica Roxb. & $\mathrm{T}$ & $\mathrm{E}$ & $\mathrm{Si}$ & $\mathrm{N}$ & 24434 \\
\hline 113 & Olea glandulifera Wall. ex. G. Don & $\mathrm{T}$ & $\mathrm{E}$ & $\mathrm{Si}$ & $\mathrm{Me}$ & 25236,24474 \\
\hline \multicolumn{7}{|l|}{ Primulaceae } \\
\hline 114 & Ardisia solanacea Roxb. & $\mathrm{S}$ & $\mathrm{E}$ & $\mathrm{Si}$ & $\mathrm{Me}$ & 25286 \\
\hline 115 & Embelia ribes Burm. f. & $\mathrm{CS}$ & $\mathrm{E}$ & $\mathrm{Si}$ & $\mathrm{Me}$ & 25811 \\
\hline 116 & Maesa indica (Roxb.) DC. & $\mathrm{T}$ & $\mathrm{E}$ & $\mathrm{Si}$ & $\mathrm{Me}$ & $25230,24454,24504$ \\
\hline 117 & Rapanea wightiana (Wall. ex DC.) Mez. & $\mathrm{T}$ & $\mathrm{E}$ & $\mathrm{Si}$ & $\mathrm{Mi}$ & 24491 \\
\hline \multicolumn{7}{|l|}{ Rhamnaceae } \\
\hline 118 & Ziziphus rugosa Lam. & $\mathrm{CS}$ & $\mathrm{E}$ & $\mathrm{Si}$ & $\mathrm{Me}$ & 24532 \\
\hline 119 & Ziziphus xylopyrus (Retz.) Willd. & $\mathrm{T}$ & $\mathrm{D}$ & $\mathrm{Si}$ & $\mathrm{N}$ & 24544 \\
\hline \multicolumn{7}{|l|}{ Rosaceae } \\
\hline 120 & Prunus ceylanica (Wight) Miq. & $\mathrm{T}$ & $\mathrm{E}$ & $\mathrm{Si}$ & $\mathrm{Me}$ & 25327 \\
\hline \multicolumn{7}{|l|}{ Rubiaceae } \\
\hline 121 & Catunaregam torulosa (Dennst.) Tirveng. & $\mathrm{T}$ & $\mathrm{D}$ & $\mathrm{Si}$ & $\mathrm{N}$ & 25806 \\
\hline 122 & Gardenia gummifera L. f. & $\mathrm{T}$ & $\mathrm{D}$ & $\mathrm{Si}$ & Mi & 25818 \\
\hline 123 & Gardenia latifolia Ait. & $\mathrm{T}$ & $\mathrm{D}$ & $\mathrm{Si}$ & Ma & $24519,24520,25301$ \\
\hline 124 & Gardenia resinifera Roth. & $\mathrm{T}$ & $\mathrm{D}$ & $\mathrm{Si}$ & $\mathrm{Me}$ & $\begin{array}{l}24386,24420,24451,25729,24524 \\
25296,25317\end{array}$ \\
\hline 125 & Haldina cordifolia (Roxb.) Ridsdale & $\mathrm{T}$ & $\mathrm{D}$ & $\mathrm{Si}$ & Ma & 24576 \\
\hline 126 & Hymenodictyon orixense (Roxb.) Mabb. & $\mathrm{T}$ & $\mathrm{D}$ & $\mathrm{Si}$ & $\mathrm{Me}$ & 24506 \\
\hline 127 & Ixora pavetta Andr. & $\mathrm{T}$ & $\mathrm{E}$ & $\mathrm{Si}$ & $\mathrm{Me}$ & $\begin{array}{l}24415,24477,24482,24515,25510 \\
24523\end{array}$ \\
\hline 128 & Mitragyna parvifolia (Roxb.) Korth. & $\mathrm{T}$ & $\mathrm{D}$ & $\mathrm{Si}$ & $\mathrm{Me}$ & 24555,25306 \\
\hline 129 & Morinda pubescens J. E. Smith & $\mathrm{T}$ & $\mathrm{D}$ & $\mathrm{Si}$ & $\mathrm{Me}$ & 24384 \\
\hline 130 & Pavetta canescens DC. & $\mathrm{T}$ & $\mathrm{D}$ & $\mathrm{Si}$ & $\mathrm{Me}$ & $24460,24501,24581$ \\
\hline 131 & Pavetta indica $\mathrm{L}$. & $\mathrm{T}$ & $\mathrm{E}$ & $\mathrm{Si}$ & $\mathrm{Me}$ & 25285 \\
\hline 132 & Psychotria nigra (Gaertn.) Alston & S & $\mathrm{E}$ & $\mathrm{Si}$ & $\mathrm{Me}$ & 24503,25521 \\
\hline 133 & Psydrax dicoccos Gaertn. & $\mathrm{T}$ & $\mathrm{E}$ & $\mathrm{Si}$ & $\mathrm{N}$ & $\begin{array}{l}24411,25228,24453,24459,24481 \\
24484,24509,25513,24540,25297\end{array}$ \\
\hline 134 & Tamilnadia uliginosa (Retz.) Tirveng. and Sastre & $\mathrm{T}$ & $\mathrm{D}$ & $\mathrm{Si}$ & $\mathrm{Me}$ & 24542,25320 \\
\hline 135 & Tarenna asiatica (L.) Kuntze & $\mathrm{S}$ & $\mathrm{E}$ & $\mathrm{Si}$ & $\mathrm{Me}$ & $\begin{array}{l}24375,24389,24422,24429,24444 \\
24473,24479,24511,25728\end{array}$ \\
\hline 136 & Wendlandia thyrsoidea (Schultes) Steud. & $\mathrm{T}$ & $\mathrm{D}$ & $\mathrm{Si}$ & $\mathrm{Me}$ & 24485,24582 \\
\hline \multicolumn{7}{|l|}{ Rutaceae } \\
\hline 137 & Aegle marmelos (L.) Correa & $\mathrm{T}$ & $\mathrm{D}$ & $\mathrm{C}$ & Mi & 25294 \\
\hline 138 & Atalantia monophylla (L.) Correa & $\mathrm{T}$ & $\mathrm{E}$ & $\mathrm{Si}$ & $\mathrm{Mi}$ & $\begin{array}{l}24373,24408,24390,24438,25516 \\
25523,24546\end{array}$ \\
\hline 139 & Euodia lunu-ankenda (Gaertn.) Merr. & $\mathrm{T}$ & $\mathrm{E}$ & $\mathrm{C}$ & $\mathrm{Me}$ & 25232 \\
\hline 140 & Naringi alata (Wall. ex Wight and Arn.) Ellis & $\mathrm{T}$ & $\mathrm{E}$ & $\mathrm{C}$ & $\mathrm{Mi}$ & 25293 \\
\hline \multicolumn{7}{|l|}{ Sabiaceae } \\
\hline 141 & Meliosma simplicifolia (Roxb.) Walp. & $\mathrm{T}$ & $\mathrm{E}$ & $\mathrm{Si}$ & $\mathrm{Ma}$ & 24468 \\
\hline \multicolumn{7}{|l|}{ Salicaceae } \\
\hline 142 & Casearia elliptica Willd. & $\mathrm{S}$ & $\mathrm{D}$ & $\mathrm{Si}$ & $\mathrm{N}$ & 24412 \\
\hline 143 & Scolopia crenata (Wight and Arn.) Clos. & $\mathrm{T}$ & $\mathrm{E}$ & $\mathrm{Si}$ & $\mathrm{N}$ & 24505 \\
\hline \multicolumn{7}{|l|}{ Santalaceae } \\
\hline 144 & Santalum album L. & $\mathrm{T}$ & $\mathrm{E}$ & $\mathrm{Si}$ & $\mathrm{Mi}$ & $24498,24548,24549$ \\
\hline
\end{tabular}


TABle 2. Continued.

\begin{tabular}{|c|c|c|c|c|c|c|}
\hline S. NO. & BOTANICAL NAME & $\begin{array}{c}\text { LIFE } \\
\text { FORM } \\
\end{array}$ & PHENOLOGY & $\begin{array}{l}\text { LEAF } \\
\text { FORM } \\
\end{array}$ & $\begin{array}{l}\text { LEAF } \\
\text { CLASS }\end{array}$ & * HIFP NO. \\
\hline \multicolumn{7}{|l|}{ Sapindaceae } \\
\hline 145 & Dodonaea viscosa (L.) Jacq. & $\mathrm{S}$ & $\mathrm{D}$ & $\mathrm{Si}$ & $\mathrm{Mi}$ & $24396,24381,25513$ \\
\hline 146 & Lepisanthes senegalensis (Juss. ex Poir.) Leenh. & $\mathrm{T}$ & $\mathrm{E}$ & $\mathrm{C}$ & $\mathrm{N}$ & 25229 \\
\hline 147 & Sapindus emarginatus Vahl & $\mathrm{T}$ & $\mathrm{D}$ & $\mathrm{C}$ & $\mathrm{N}$ & 24410,25291 \\
\hline 148 & Schleichera oleosa (Lour.) Oken & $\mathrm{T}$ & $\mathrm{D}$ & $\mathrm{C}$ & $\mathrm{Me}$ & 25288,24541 \\
\hline \multicolumn{7}{|l|}{ Sapotaceae } \\
\hline 149 & Madhuca longifolia var. latifolia (Roxb.) A. Chev. & $\mathrm{T}$ & $\mathrm{D}$ & $\mathrm{Si}$ & $\mathrm{Me}$ & 24402,24510 \\
\hline 150 & Manilkara hexandra (Roxb.) Dubard & $\mathrm{T}$ & $\mathrm{E}$ & $\mathrm{Si}$ & $\mathrm{N}$ & $24368,24377,24431,25295$ \\
\hline 151 & Mimusops elengi L. & $\mathrm{T}$ & $\mathrm{E}$ & $\mathrm{Si}$ & $\mathrm{Me}$ & 24516,25515 \\
\hline \multicolumn{7}{|l|}{ Symplocaceae } \\
\hline 152 & Symplocos cochinchinensis (Lour.) Moore & $\mathrm{T}$ & $\mathrm{E}$ & $\mathrm{Si}$ & $\mathrm{Me}$ & $25233,24455,24488$ \\
\hline \multicolumn{7}{|l|}{ Ulmaceae } \\
\hline 153 & Celtis philippensis Blanco & $\mathrm{T}$ & $\mathrm{E}$ & $\mathrm{Si}$ & $\mathrm{Me}$ & 25459 \\
\hline 155 & Holoptelea integrifolia (Roxb.) Planch. & $\mathrm{T}$ & $\mathrm{D}$ & $\mathrm{Si}$ & $\mathrm{Me}$ & $24433,24440,24441,25511,24579$ \\
\hline \multicolumn{7}{|l|}{ Verbenaceae } \\
\hline 156 & Callicarpa tomentosa (L.) Murr. & $\mathrm{T}$ & $\mathrm{D}$ & $\mathrm{Si}$ & $\mathrm{Me}$ & 25810 \\
\hline 157 & Gmelina arborea Roxb. & $\mathrm{T}$ & $\mathrm{D}$ & $\mathrm{Si}$ & $\mathrm{Me}$ & 24388 \\
\hline 158 & $\begin{array}{l}\text { Premna latifolia Roxb. var. mollissima (Roth) C. } \\
\text { B. Clarke }\end{array}$ & $\mathrm{T}$ & $\mathrm{D}$ & $\mathrm{Si}$ & $\mathrm{Me}$ & 25334,25300 \\
\hline 159 & Premna tomentosa Roxb. & $\mathrm{T}$ & $\mathrm{D}$ & $\mathrm{Si}$ & $\mathrm{Me}$ & 24535 \\
\hline
\end{tabular}

In the present study, different leaf sizes were observed within the same species (Table 3). We have assigned leaf classes to these heterophyllous leaf species based on the dominant leaf class exhibited by maximum number of individuals. It is well known that environmental conditions can lead to heterophylly i.e., two or more distinct leaf forms in the same species (Vaughan and Wiehe 1939). Leaf size variation among ecologically similar species and within species may be associated with their function (Ackerly and Reich 1999).

TABLE 3. Heterophylly shown by some plant species of Eastern Ghats (Data are means \pm standard deviations (no of individuals)).

\begin{tabular}{llll}
\hline SPECIES & MICROPHYLL/NOTOPHYLL $\mathbf{( M M}^{2}$ ) & SPECIES & NOTOPHYLL/MESOPHYLL (MM ${ }^{2}$ ) \\
\hline Atalantia monophylla & $1637.38 \pm 984.17(8)$ & Anogeissus latifolia & $3966.25 \pm 1477.22(5)$ \\
Drypetes sepiaria & $1745.33 \pm 671.65(9)$ & Diospyros melanoxylon & $5886.06 \pm 1547.05(4)$ \\
Holoptelea integrifolia & $4302.21 \pm 1487.84(6)$ & Holoptelea integrifolia & $4302.21 \pm 1487.84(6)$ \\
Memecylon umbellatum & $1724.48 \pm 714.65(10)$ & Ixora pavetta & $6591.75 \pm 2040.34(8)$ \\
& & Mallotus philippensis & $6651.25 \pm 2875.8(5)$ \\
& & Psydrax dicoccus & $4608.83 \pm 880.71(12)$ \\
& & Syzygium cumini & $4715.32 \pm 1519.37(11)$ \\
\hline
\end{tabular}

Of the total 159 taxa, 83 are evergreen and 76 are deciduous. Here, the evergreen or deciduous nature is based on the foliar phenology of the plant and irrespective of its occurrence in different vegetation types. Further we do not differentiate a brevi-deciduous category. Phenological patterns of tropical forest trees are diverse and complex and dependent on equally complex factors (Bawa and Hedley 1990; Williams-Linera 1997) such as precipitation, tree water status (Reich and Borchert 1982; Borchert 1994), irradiance (Wright and van Schaik 1994) and temperature (Ashton et al. 1988; Tutin and Fernandez 1993). The robust pattern appears to be that deciduous trees lose their leaves in a particular season but evergreen trees drop leaves throughout the year (Williams-Linera 1997). The distinction between deciduous and evergreen habit is not always straight forward (Thomas 2000). For instance, Shorea robusta is considered as an evergreen species (Krishnaswamy and Mathauda 1954) but this species was referred as tardily deciduous (Li et al. 1994), deciduous (Tiwari 1995) and observed leafless for about 8 days in Hathinala forest area of Vindhyan region (Kushwaha and Singh 2005). The degree of gradient of deciduousness is ascertained only by finer field observations (Singh and Kushwaha 2005). Not surprisingly, we found that our study assigned a different phenology to 15 species (Table 4) in comparison with Pragasan and Parthasarathy (2009). Of these, 6 that were assigned evergreen by us were characterized brevideciduous in the earlier study. However, Haldina cordifolia, observed to be deciduous in the present study was marked as evergreen by Pragasan and Parthasarathy (2009); Newton (1988) in central Indian highlands, Mishra et al. (2006) in Similipal biosphere reserve, Orissa and Desai and Patel (2010) in Satlasana range forest, north Gujarat observed H. cordifolia as deciduous. Likewise, in Ficus drupacea, leaf flushing was observed from January to midMay in one season whereas the same was observed during September to December in the next season in the same site in Coorg of Western Ghats (Patel, 1997) and the same species has been marked as brevi-deciduous by Pragasan 
and Parthasarathy (2009). The added value of the present study is that it draws attention to the fact that long-term observations at a finer spatial scale are particularly important in deciduous forests.

In the present study, leaf area is significantly correlated with the altitude (two-tailed p-value is 0.002 where alpha is 0.05). Leaf area increased with increasing altitude. Leaf area is not significantly correlated with mean annual temperature (MAT) and mean annual precipitation (MAP). The narrow difference in temperature and precipitation has no effect on the leaf size. The dominant mesophyllous species are highly adaptive within this short range of MAT and MAP as seen in Amazonian rainforest trees where only megaphyllous trees correlated significantly with environmental factors (Malhado et al. 2009). Specific leaf area, stomatal length and index increased with increasing altitude below $2800 \mathrm{~m}$, but decreased with increasing altitude above $2800 \mathrm{~m}$ ( $\mathrm{Li}$ et al. 2006). Royer et al. (2008) suggests that leaf size is not sensitive to MAT and MAP and this is due to wind speed and water availability which underpin these elevation patterns. But still leaf size may be used to know major fluctuations in, for example, mean annual biotemperature in regions such as Costa Rica, Barro Colorado Island, Panama (tropical) and 17 eastern North American sites (temperate) (Dolph and Dilcher 1980a; Royer et al. 2005).

Earlier it was thought that leaf size cannot be used to identify specific life zone or climates in either extant or fossil floras (Dolph and Dilcher 1980b) but recent studies suggest that leaf sizes in modern vegetation accurately predict MAP and can be used in the prediction of climate of fossil leaf assemblages (Wilf et al. 1998). Wolfe (1971, 1990, 1995) and Wolfe and Upchurch (1987a, b) have undertaken a comprehensive analysis of palaeofloristic data bearing on the interpretation of Late Cretaceous climates using the extant leaf physiognomy study. Correspondence analysis of Dicotyledones leaf physiognomy of modern vegetational samples from a wide range of environments indicates that more than $70 \%$ physiognomic variation corresponds to water or temperature factors, or both. Wolfe (1990) applied this climate-leaf analysis multivariate program (CLAMP) to leaf assemblages from the Cretaceous/Tertiary boundary for the inference of climate characteristics from physiognomic analyses.

South American models for climate reconstruction indicate that MAT - leaf morphology correlation equations based on Northern Hemisphere or Australian data do not accurately predict the MAT of South American sites but the use of tropical south America data itself may help in predicting the MAT of low temperature and high elevation modern sites as well as fossil sites (Kowalski 2002). In this sense, the data presented in this paper can be used to predict the climate variables using leaf size with additional inputs from various other regions of India. The prediction of MAT by analyzing leaf size is largely dependent on the habitat of the plant species (Burnham et al. 2001). It was observed that leaf size tends to increase from colder to warmer environments in humid sites (Grubb 1977; Tanner 1980; Niinemets et al. 2007); however, in the present study the narrow range of temperatures does not allow us to discern any predictable relationship between leaf size and MAT.

The present study standardized the methodology for the leaf trait measurements in the tropical region and also initiated a baseline data on leaf area measurements (Supporting Information). The quantitative trait measurements from the Eastern Ghats do add a distinct dimension to the field of functional ecology: even within the narrow range of temperature and precipitation in the

TABLE 4. Foliar phenology assignments: Pragasan and Parthasarathy (2009) and present study.

\begin{tabular}{|c|c|c|c|}
\hline S. NO. & BOTANICAL NAME* & $\begin{array}{c}\text { PHENOLOGY } \\
\text { (PRESENT STUDY) }\end{array}$ & $\begin{array}{c}\text { PHENOLOGY } \\
\text { (PRAGASAN AND PARTHASARATHY, 2009) }\end{array}$ \\
\hline \multicolumn{4}{|c|}{ Anacardiaceae } \\
\hline 1 & Buchanania lanzan Spreng. & $\mathrm{E}$ & $\mathrm{D}$ \\
\hline 2 & Mangifera indica $\mathrm{L}$. & $\mathrm{E}$ & $\mathrm{BD}$ \\
\hline \multicolumn{4}{|l|}{ Araliaceae } \\
\hline 3 & Schefflera stellata (Gaertn.) Harms & $\mathrm{E}$ & $\mathrm{D}$ \\
\hline \multicolumn{4}{|l|}{ Ebenaceae } \\
\hline 4 & Diospyros melanoxylon Roxb. & $\mathrm{D}$ & $\mathrm{E}$ \\
\hline \multicolumn{4}{|l|}{ Malvaceae } \\
\hline 5 & Grewia orbiculata Rottl. & $\mathrm{D}$ & $\mathrm{E}$ \\
\hline 6 & Helicteres isora L. & $\mathrm{D}$ & $\mathrm{E}$ \\
\hline 7 & Pterospermum canescens Roxb. & $\mathrm{E}$ & $\mathrm{BD}$ \\
\hline 8 & Pterospermum reticulatum Wight and Arn. & $\mathrm{E}$ & $\mathrm{D}$ \\
\hline \multicolumn{4}{|l|}{ Moraceae } \\
\hline 10 & Ficus drupacea Thunb. & $\mathrm{E}$ & $\mathrm{BD}$ \\
\hline 11 & Ficus mollis Vahl & $\mathrm{E}$ & $\mathrm{BD}$ \\
\hline 12 & Ficus virens Ait. & $\mathrm{E}$ & BD \\
\hline \multicolumn{4}{|l|}{ Rubiaceae } \\
\hline 13 & Haldina cordifolia (Roxb.) Ridsdale & $\mathrm{D}$ & $\mathrm{E}$ \\
\hline \multicolumn{4}{|c|}{ Verbenaceae } \\
\hline 14 & Premna latifolia Roxb. var. mollissima (Roth) C. B. Clarke & $\mathrm{D}$ & $\mathrm{E}$ \\
\hline 15 & Premna tomentosa Roxb. & $\mathrm{D}$ & $\mathrm{E}$ \\
\hline
\end{tabular}

The species reported here correspond to those with different attributions in the two studies 
study area, heterophylly is conclusively recorded in 10 species; further, leaf area is significantly correlated with altitude, showing that by extending such studies wider, a definite relationship with climatic parameters, such as precipitation or water availability may also be established. Using information from and comparing with existing checklists from this region has also yielded interesting results and pointers for further research: the need for spatially finer long term monitoring studies to reach definitive conclusions about phenology in deciduous forests and the importance of establishing quantitative measurements for species with compound leaves that are more prevalent in this study area characterized by higher temperatures and lower mean annual precipitation, among them. Leaf area measurements and corresponding climatic data derived from the WorldClim data set (Hijmans et al. 2005) are provided in Supporting Information contributing to the CLAMP data base.

In the face of the present crises in the conservation and management of tropical forests, particularly their fragmentation, assessments of functional diversity as much as species diversity are important. The present study does indicate this, though there is potential for refinements and applicability in the plant functional classification; critically, wider sampling of sites is needed to increase number of species, geographic and climatic coverage and the number of leaf economic variables (e.g., leaf lifespan, nutrient content).
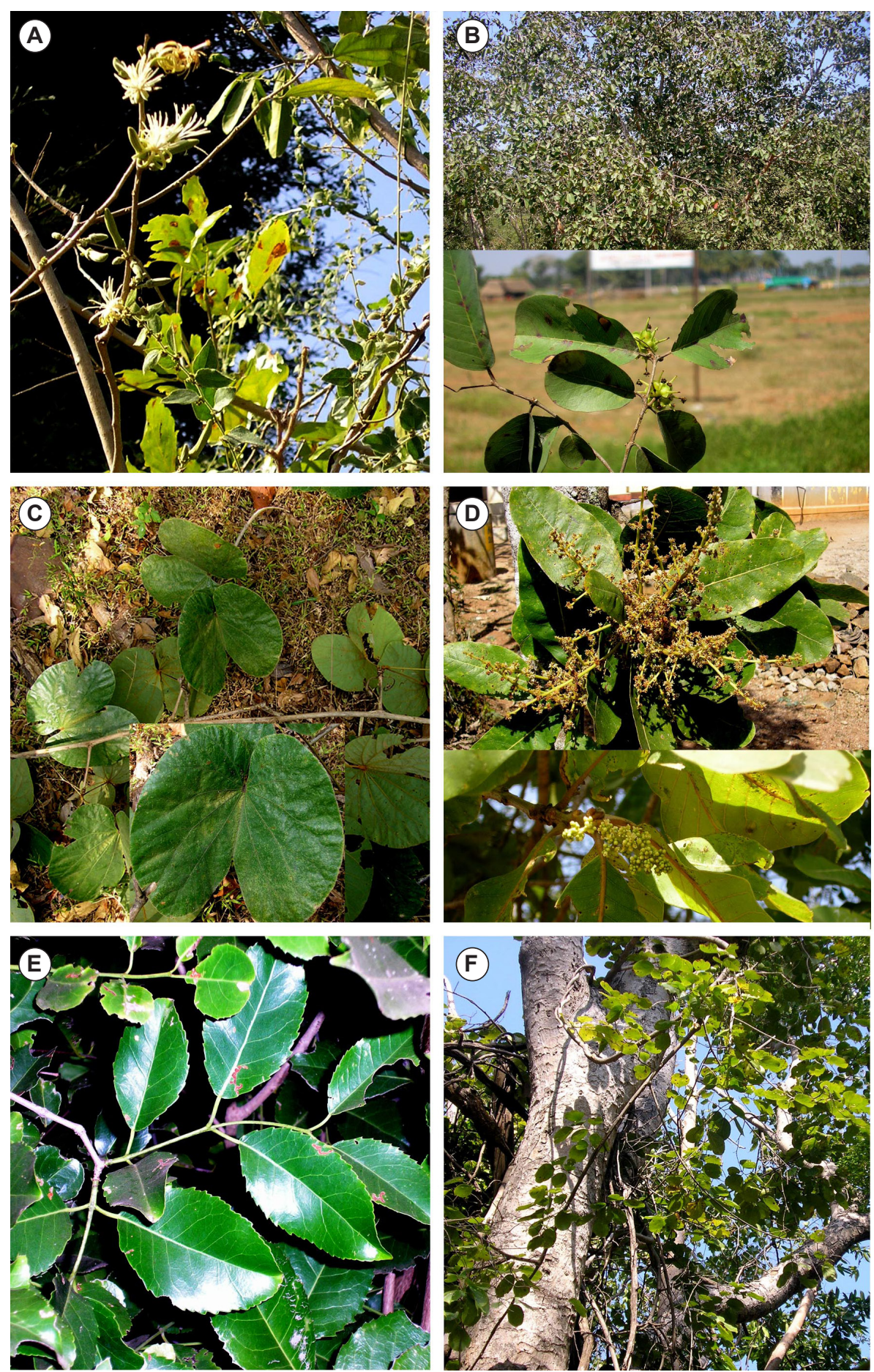

Figure 2. a) Alangium salvifolium (Cornaceae); b) Anogeissus latifolia (Combretaceae); c) Bauhinia vahlii (Fabaceae); d) Buchanania lanzan (Anacardiaceae); e) Cassine glauca (Celastraceae); f) Celastrus paniculatus (Celastraceae). Photos by Stephen A. 

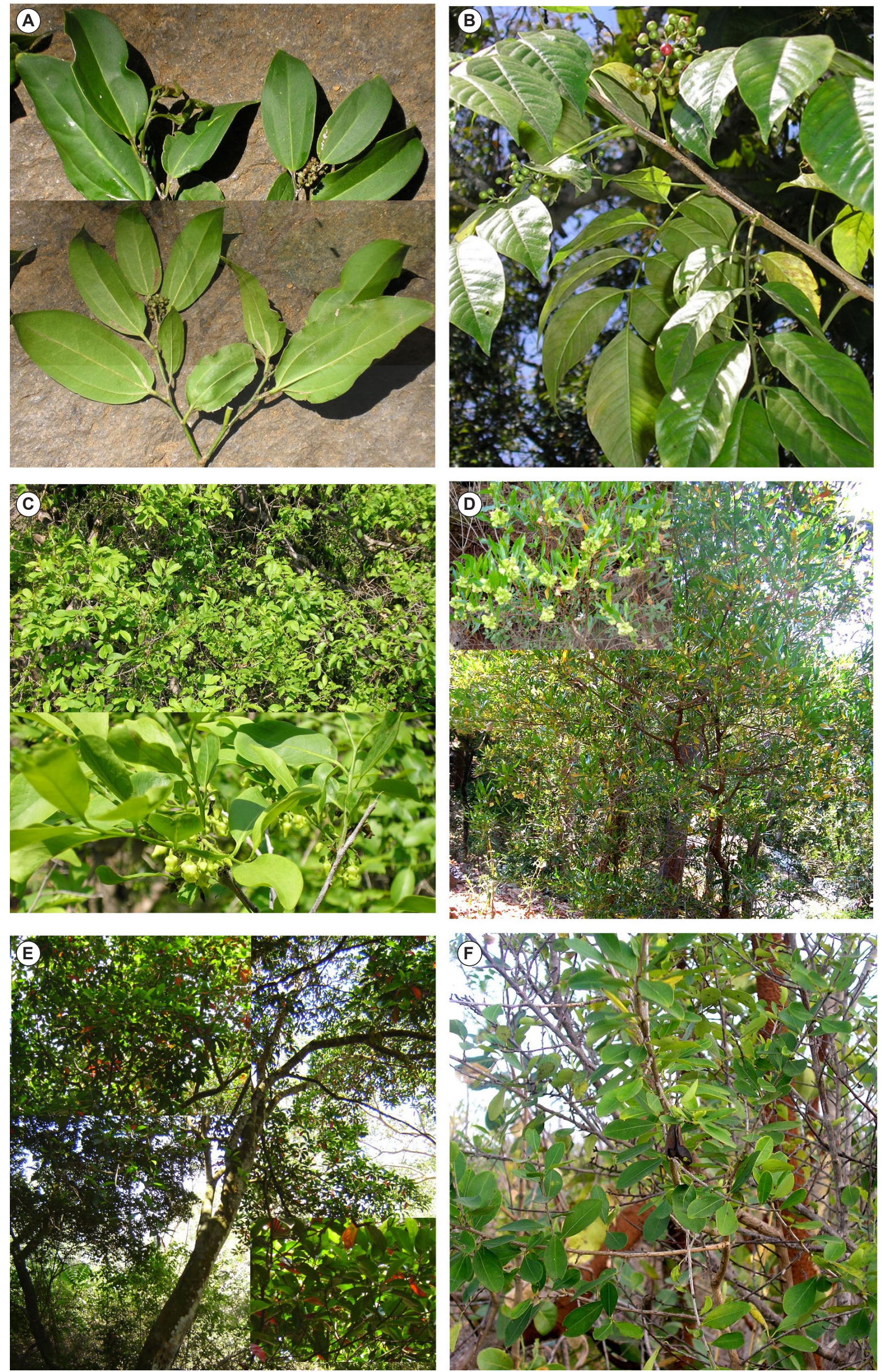

Figure 3. a) Celtis philippensis var. wightii (Ulmaceae); b) Cipadessa baccifera (Meliaceae); c) Diospyros montana (Ebenaceae); d) Dodonaea viscosa (Sapindaceae); e) Elaeocarpus serratus (Elaeocarpaceae); f) Erythroxylum monogynum (Erythroxylaceae). Photos by Stephen A. 



FiguRE 4. a) Ficus amplissima (Moraceae); b) Ficus arnottiana (Moraceae); c) Flueggea leucopyrus (Euphorbiaceae); d) Gardenia latifolia (Rubiaceae); e) Gardenia resinifera (Rubiaceae); f) Glochidion ellipticum (Euphorbiaceae). Photos by Stephen A. 

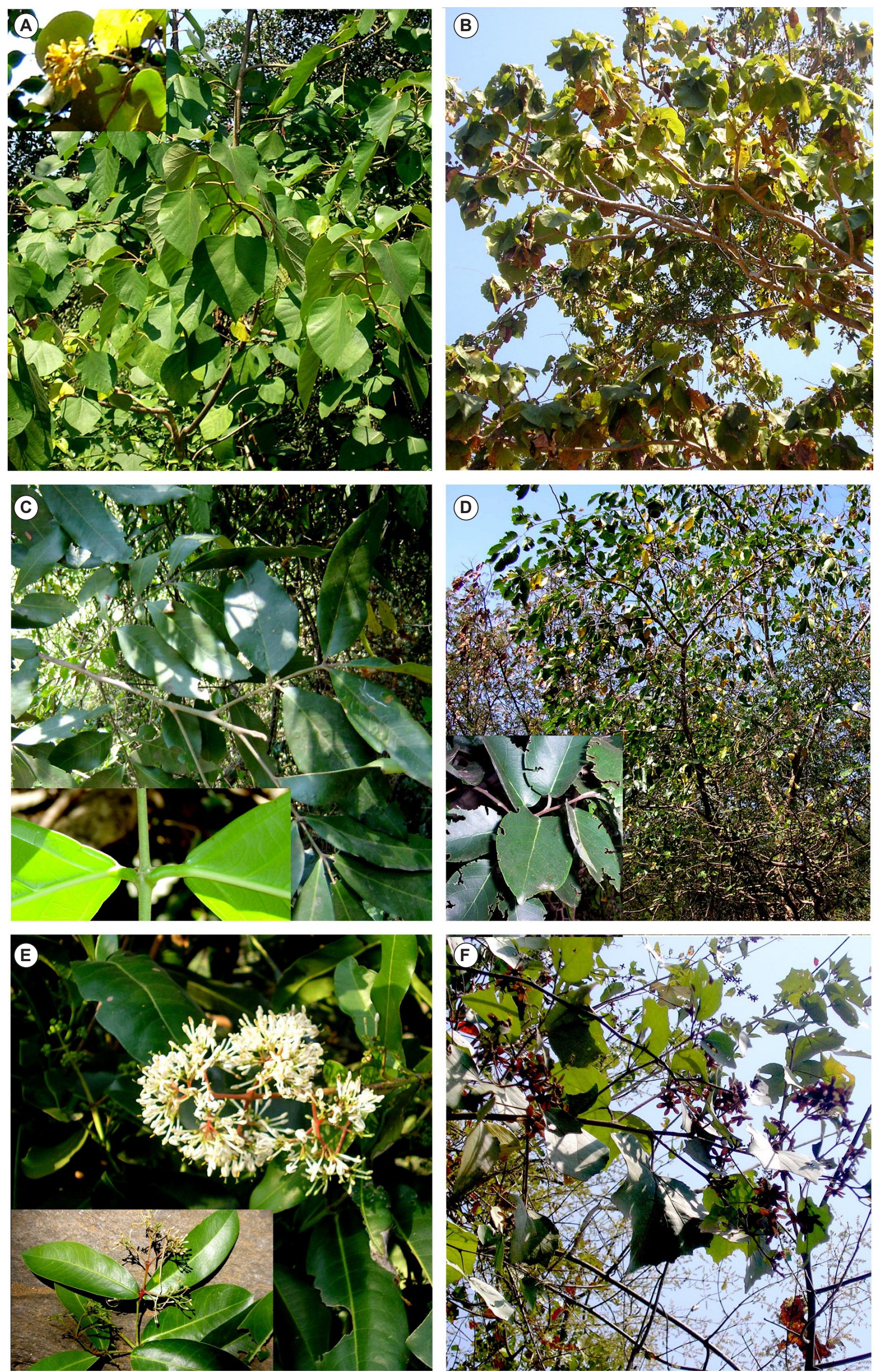

FiguRE 5. a) Gmelina arborea (Verbenaceae); b) Haldina cordifolia (Rubiaceae); c) Hiptage benghalensis (Malpighiaceae); d) Holoptelea integrifolia (Ulmaceae); e) Ixora pavetta (Rubiaceae); f) Kydia calycina (Malvaceae). Photos by Stephen A. 

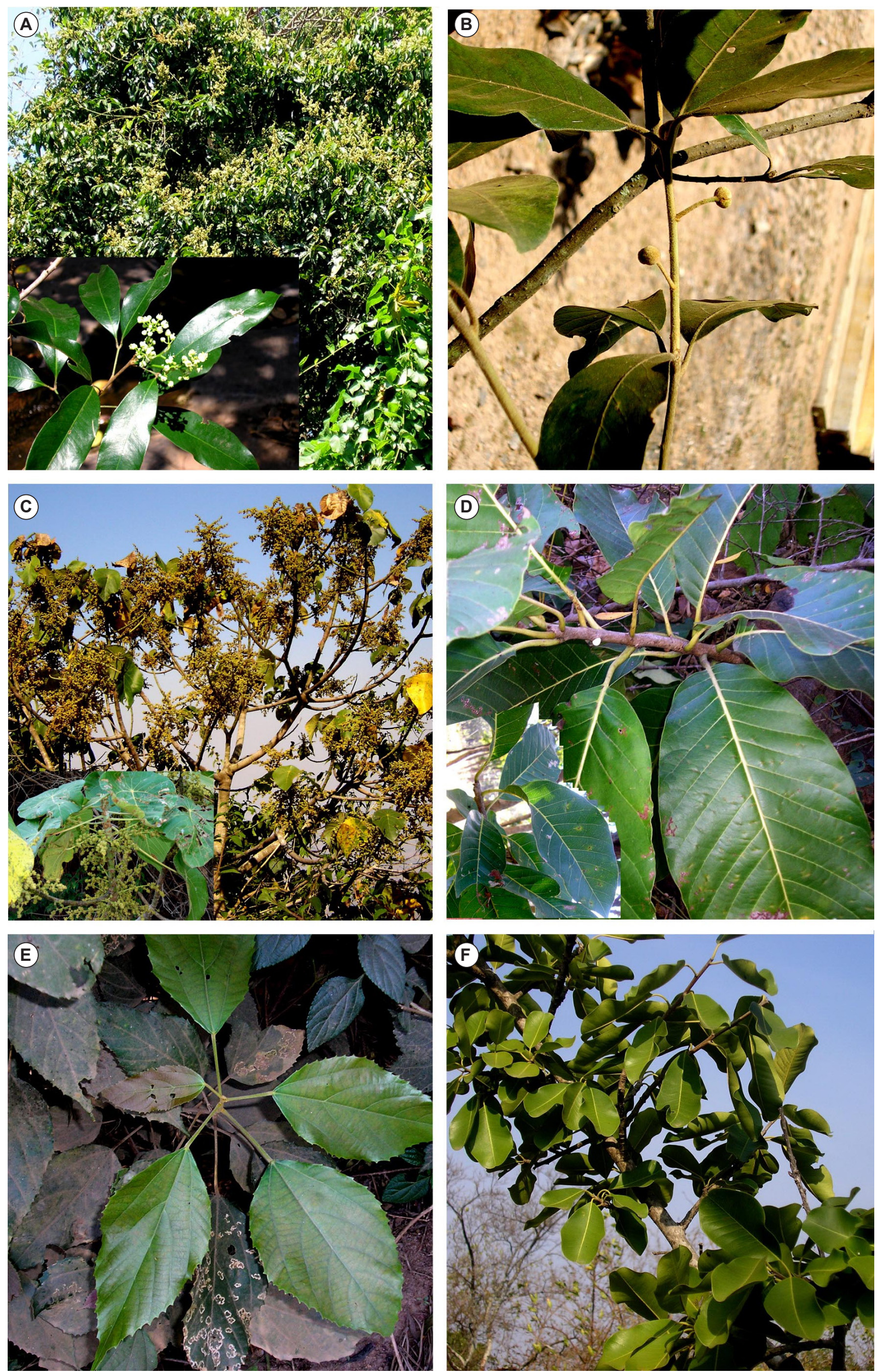

FiguRE 6. a) Lepisanthes senegalensis (Sapindaceae); b) Litsea deccanensis (Lauraceae); c) Macaranga peltata (Euphorbiaceae); d) Madhuca longifolia var. latifolia (Sapotaceae); e) Mallotus philippensis (Euphorbiaceae); f) Manilkara hexandra (Sapotaceae). Photos by Stephen A. 

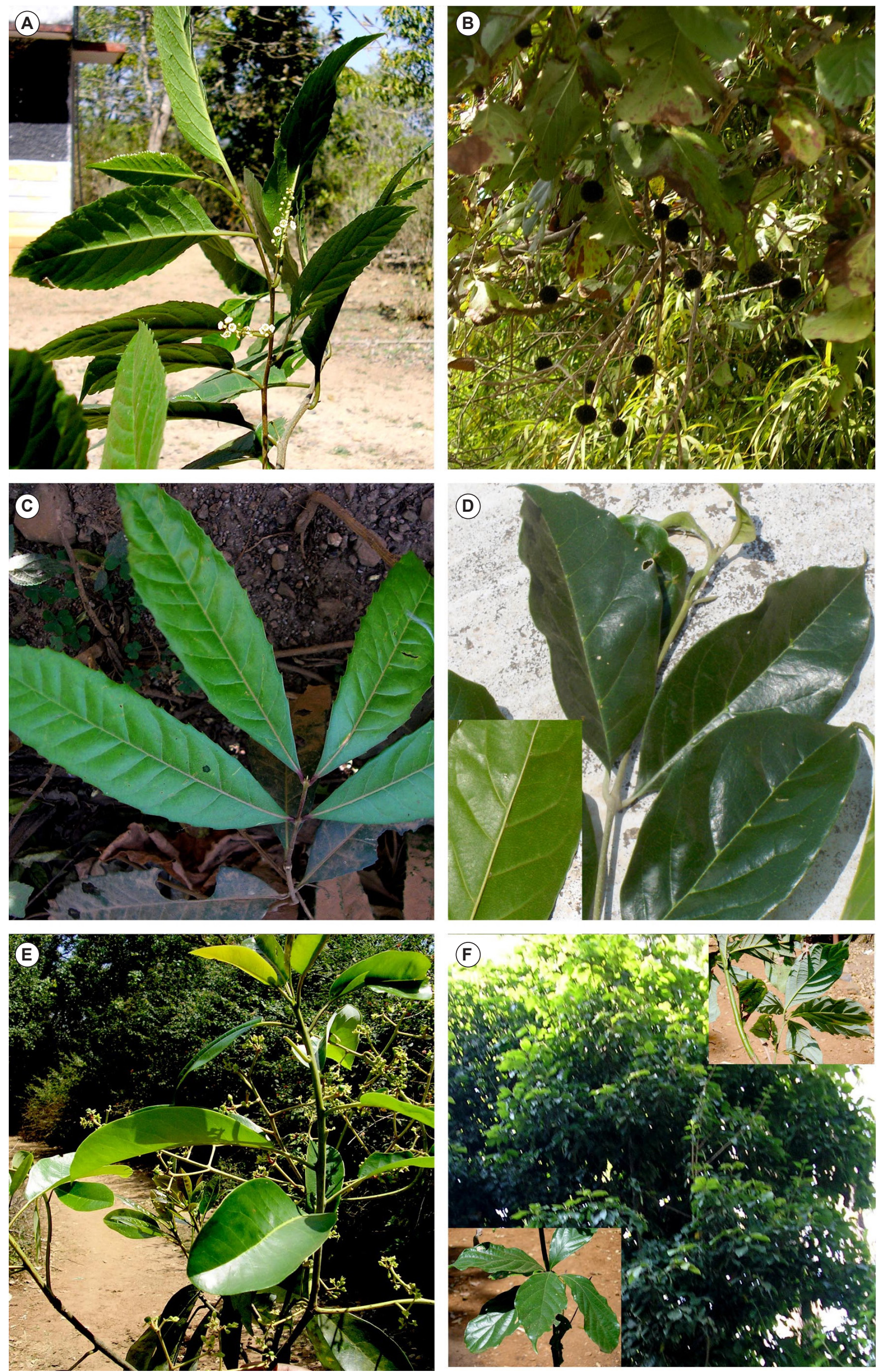

Figure 7. a) Maesa indica (Myrsinaceae); b) Mitragyna parvifolia (Rubiaceae); c) Olea dioica (Oleaceae); d) Olea glandulifera (Oleaceae); e) Persea macrantha (Lauraceae); f) Phoebe paniculata (Lauraceae). Photos by Stephen A. 

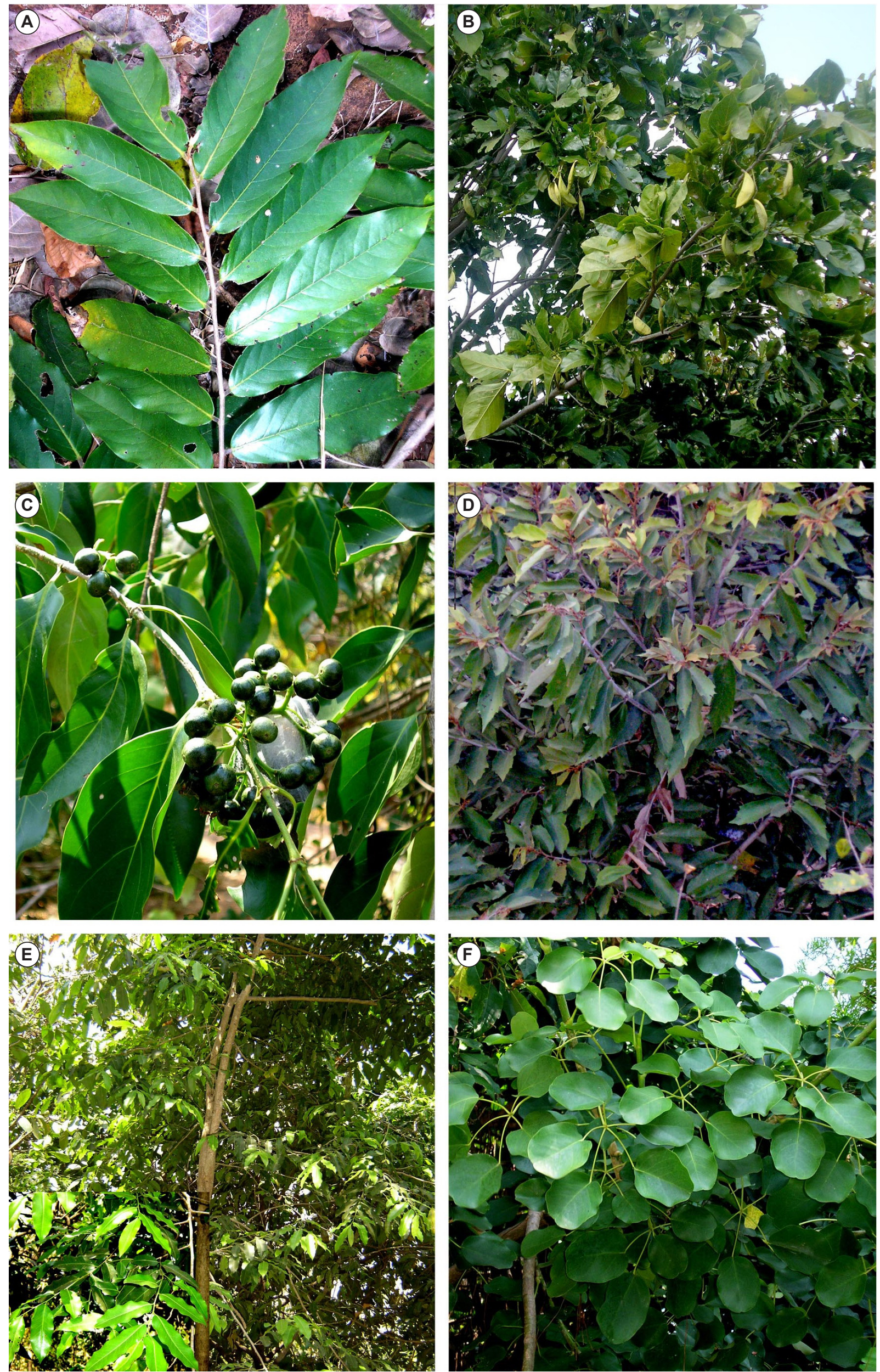

Figure 8. a) Polyalthia cerasoides (Annonaceae); b) Pongamia pinnata (Fabaceae); c) Psydrax dicoccos (Rubiaceae); d) Pterospermum canescens (Malvaceae); e) Putranjiva roxburghii (Euphorbiaceae); f) Schefflera stellata (Araliaceae). Photos by Stephen A. 

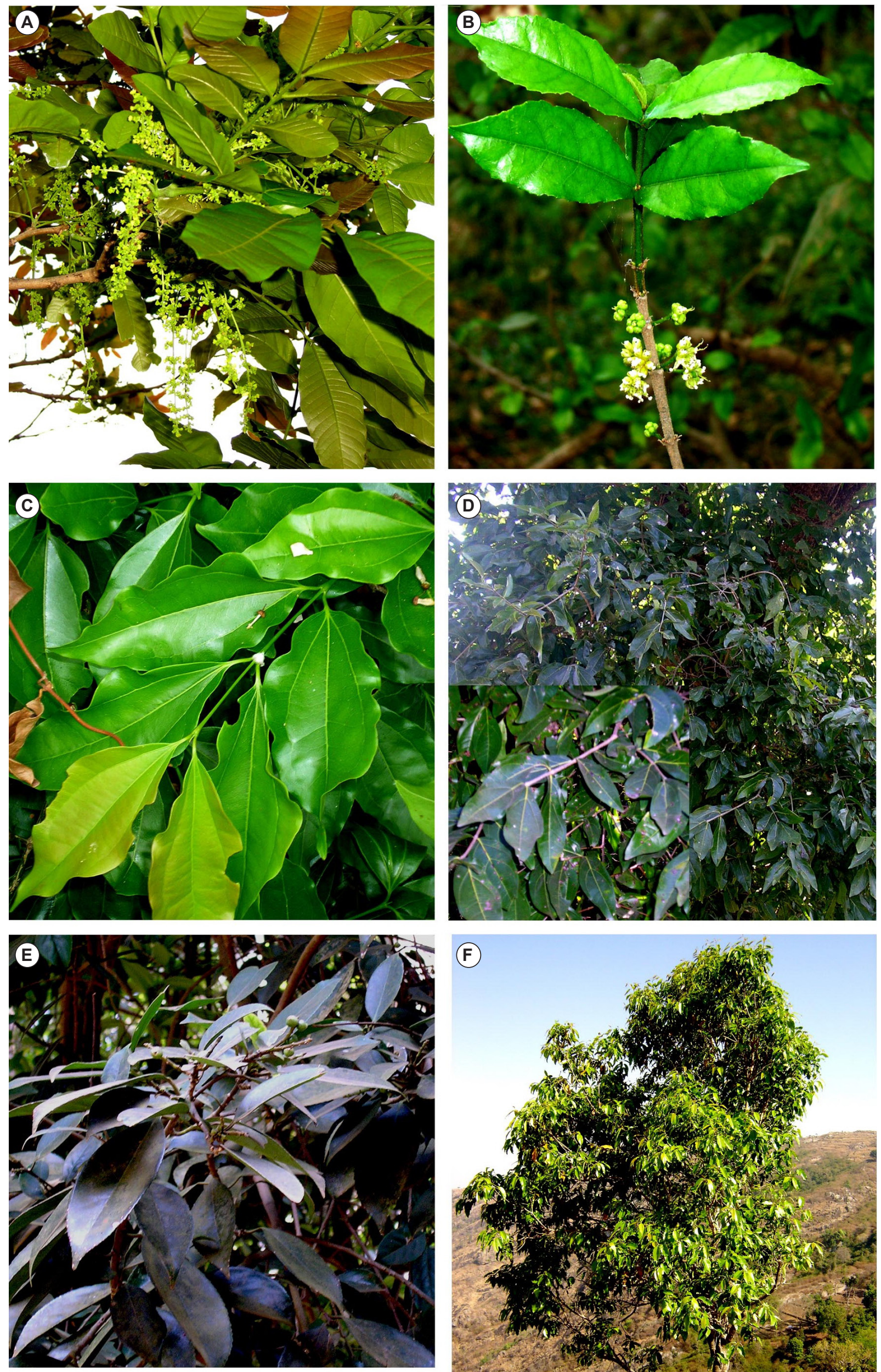

FiguRE 9. a) Schleichera oleosa (Sapindaceae); b) Streblus asper (Moraceae); c) Strychnos nux-vomica (Loganiaceae); d) Strychnos potatorum (Loganiaceae); e) Symplocos cochinchinensis (Symplocaceae); f) Syzygium cumini (Myrtaceae). Photos by Stephen A. 

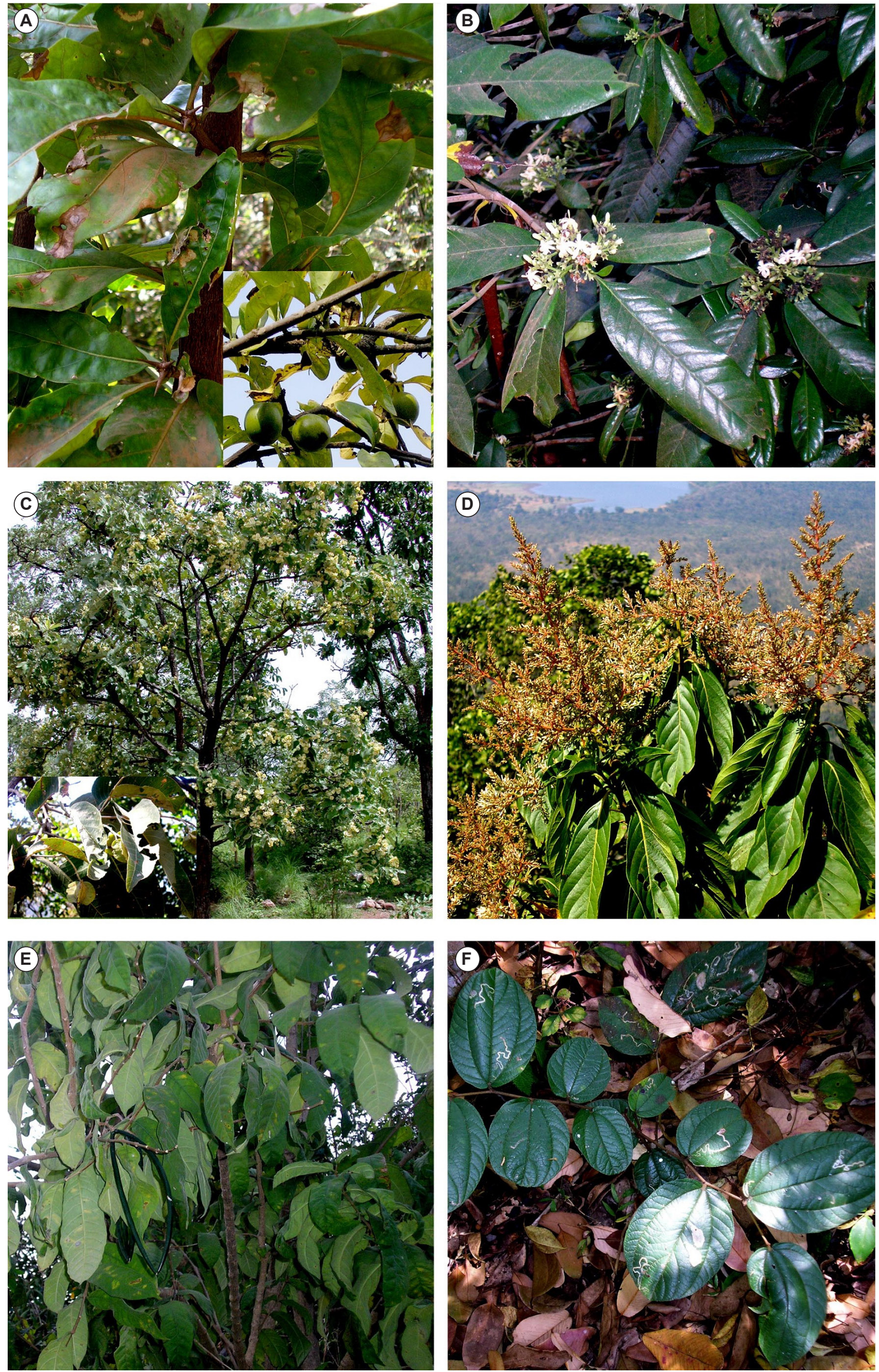

Figure 10. a) Tamilnadia uliginosa (Rubiaceae); b) Tarenna asiatica (Rubiaceae); c) Terminalia alata (Combretaceae); d) Wendlandia thyrsoidea (Rubiaceae); e) Wrightia tinctoria (Apocynaceae); f) Ziziphus rugosa (Rhamnaceae). Photos by Stephen A. 
Acknowledgments: We thank the National Remote Sensing Centre (NRSC, formerly NRSA), Hyderabad, India for financial support through a project and for the Land Use/Land Cover information from Natural Resources Census Project of NRSC, used in our study area map. We are thankful to all the personnel of Headquarters and Divisional offices of forest departments of Andhra Pradesh and Tamil Nadu - especially Mr. Thulsi Rao, Assistant Conservator of Forests, Andhra Pradesh for various logistics especially accommodation at the field station in Nallamalais. We acknowledge the help of Mr. G. Orukaimani, Mr. R. Ponnuchamy, Mr. K. Adimoolam and Mr. N. Barathan, IFP in the field and lab work and Mr. H. Ram Mohan with data analyses. We thank Mr. G. Muthusankar and Mr. R. Sivarajan (IFP) for preparing the study area map. Several people at the IFP took time to give critical feedbacks that greatly helped improve our manuscript and we are grateful to all of them: Dr. Dario De Franceschi (MNHS), Dr. Vincent Bonhomme, Dr. N. Ayyappan and Mr. S. Prasad. The authors would like to thank two anonymous reviewers for their valuable comments and suggestions to improve the quality of the paper.

\section{Literature Cited}

Ackerly, D.D. and P.B. Reich. 1999. Convergence and correlations among leaf size and function in seed plants: a comparative test using independent contrasts. American Journal of Botany 86(9): 12721281.

APG (The Angiosperm Phylogeny Group) III. 2009. An update of the angiosperm phylogeny group classification for the orders and families of flowering plants: APG III. Botanical Journal of the Linnean Society 161: 105-121.

Ashton, P.S., T.G. Givinish and S. Appanah. 1988. Staggered flowering in the Dipterocarpaceae: new insights into floral induction and evolution of mast fruiting in the aseasonal tropics. American Naturalist 132: 4466.

Balaguru, B., S.J. Britto, N. Nagamurugan, D. Natarajan and S. Soosairaj. 2006. Identifying conservation priority zones for effective management of tropical forests in Eastern Ghats of India. Biodiversity and Conservation 15:1529-1543.

Barboni, D., S.P. Harrison, P. J. Bartlein, G. Jalut, M. New, I. C. Prentice, M.-F. Sanchez- Goni, A. Spessa, B. Davis and A.C. Stevenson, 2004. Relationship between plant traits and climate in the Mediterranean region: A pollen data analysis. Journal of Vegetation Science 15: 635646.

Bawa, K.S. and M. Hadley. 1990. Reproductive ecology of tropical forest plants. MAB Series, Vol. 7. London: UNESCO. 421 p.

Blasco, F. 1979. Les territoires biogéographiques du sous-continent Indien; p. 25-30 In P. Cassagnau (ed). Paléogéographie et Biogéographie de l'Himalaya et du Sous-Continent Indien. Toulouse: Université Paul-Sabatier.

Bonan, G.B., S. Levis, L. Kergoat and K.W. Oleson. 2002. Landscapes as patches of plant functional types: An integrating concept for climate and ecosystem models. Global Biogeochemical Cycles 16(2): 1021, doi:10.1029/2000GB001360.

Borchert, R. 1994. Soil and stem water storage determine phenology and distribution of tropical dry forest trees. Ecology 75: 1437-144.

Burnham, R.J., N.C.A. Pitman, K.R. Johnson and P. Wilf. 2001. Habitatrelated error in estimating temperatures from leaf margins in a humid tropical forest. American Journal of Botany 88(6): 1096-1102.

Cain, S.A. and G.M. de Oliveira Castro. 1959. Manual of vegetation analysis. New York: Harper and Brothers Publishers. 325 p.

Cain, S.A., G. M. de Oliveira Castro, J. M. Pires, and N.T. da Silva. 1956. Source application of some phytosociological techniques to Brazilian rain forest. American Journal of Botany 43 (10): 911-941.

Champion, H.G. and S.K. Seth. 1968. A revised survey of the forest types of India. Delhi: The Manager of Publications, Government of India. $404 \mathrm{p}$.

Chatterjee, D. 1939. Studies on the endemic flora of India and Burma. Journal of the Royal Asiatic Society of Bengal (Science) 5:19-67.

Cornelissen, J.H.C., S. Lavorel, E. Garnier, S. Diaz, N. Buchmann, D.E. Gurvich, P. B. Reich, H. ter Steege, H. D. Morgan, M.G.A. van der Heijden, J.G. Pausas and H. Poorter. 2003. A handbook of protocols for standardised and easy measurement of plant functional traits worldwide. Australian Journal of Botany 52: 335-380.

Desai, P.B. and N. K. Patel. 2010. Phenological study of trees species of Satlasana range forest (North Gujarat). Life sciences Leaflets 3:41-46.

Dobmeier, C. and M.M. Raith. 2003. Crustal architecture and evolution of the Eastern Ghats belt and adjacent regions of India; p. 145168 In A. Yoshida, B.F. Windley and S. Dasgupta (ed.). Proterozoic east Gondwana: Supercontinenet assembly and breakup. London: Geological Society of London, Special Publication, 206.

Dolph, G.E. and D.L. Dilcher. 1980a. Variation in leaf size with respect to climate in Costa Rica. Biotropica 12 (2): 91-99.

Dolph, G.E. and D.L. Dilcher. 1980b. Variation in leaf size with respect to climate in the tropics of the Western Hemisphere. Bulletin of the
Torrey Botanical Club 107 (2): 154-162.

Gamble, J.S. and C.E.C. Fischer. 1915-1935. Flora of the presidency of Madras. London: Adlard \& Son. vols. I to III. 2017 p.

Garnier, E., B. Shipley, C. Roumet, and G. Laurent. 2001. A standardized protocol for the determination of specific leaf area and leaf dry matter content. Functional Ecology 15: 688-695.

Gillison, A. 2002. A generic, computer-assisted method for rapid vegetation classification and survey: tropical and temperate case studies. Conservation Ecology 6(2): 3. [online] URL: http://www. consecol.org/vol6/iss2/art3

Grubb P.J. 1977. Control of forest growth and distribution on wet tropical mountains: with special reference to mineral nutrition. Annual Review of Ecology and Systematics 8: 83-107.

Henry, A.N., G.R. Kumari, and V. Chitra. (ed). 1987. Flora of Tamil Nadu, India, Series I: Analysis, Vol. 2. Coimbatore: Botanical Survey of India. $260 \mathrm{p}$.

Hijmans, R.J., S.E. Cameron, J.L. Parra, P.G. Jones and A. Jarvis. 2005. Very high resolution interpolated climate surfaces for global land areas. International Journal of Climatology 25: 1965-1978.

Hooker, J.D. 1872-1879. Flora of British India. London: L. Reeve \& Co. part 1-7. $5569 \mathrm{p}$.

Hooper, D.U. and P.M. Vitousek. 1997. The effects of plant composition and diversity on ecosystem processes. Science 277: 1302-1305.

Hooper, D.U. and P.M. Vitousek. 1998. Effects of plant composition and diversity on nutrient cycling. Ecological Monographs 68: 121-149.

Hooper, D.U., F.S. Chapin III, J.J. Ewel, A. Hector, P. Inchausti, S. Lavorel, J.H. Lawton, D.M. Lodge, M. Loreau, S. Naeem, B. Schmid, H. Setälä, A.J. Symstad, J. Vandermeer and D.A. Wardle. 2005. Effects of biodiversity on ecosystem functioning: a consensus of current knowledge. Ecological Monographs 75 (1): 3-35.

Jayakumar, S., D.I. Arockiasamy, and S.J. Britto. 2002. Conserving forests in the Eastern Ghats through remote sensing and GIS - A case study in Kolli hills. Current Science 82(10): 1259-1267.

Kadavul, K. and N. Parthasarathy. 1999a. Plant biodiversity and conservation of tropical semi-evergreen forest in the Shervarayan hills of Eastern Ghats, India. Biodiversity and Conservation 8: 421439.

Kadavul, K. and N. Parthasarathy. 1999b. Structure and composition of woody species in tropical semi-evergreen forest of Kalrayan hills, Eastern Ghats, India. Tropical Ecology 40: 247-260.

Kowalski, E.A. 2002. Mean annual temperature estimation based on leaf morphology: a test from tropical South America. Palaeogeography, Palaeoclimatology, Palaeoecology 188: 141-165.

Krishnan, M.S. 1961. The structure and tectonic history of India (revised). Geological Survey of India Memoirs 81: 1-137.

Krishnan, M.S. 1974. Geology; p. 60-98 In M.S. Mani (ed.). Ecology and biogeography in India. The Hague: Dr. W. Junk Publications.

Krishnaswamy, V.S. and G.S. Mathauda. 1954. Phenological behaviour of a few forest spp. at New Forest, Dehra Dun. Indian Forester 80: 3-4.

Kushwaha, C.P. and K.P. Singh. 2005. Diversity of leaf phenology in a tropical deciduous forest in India. Journal of Tropical Ecology 21: 47 56.

Landsberg, J., S. Lavorel and J. Stol. 1999. Grazing response groups among understorey plants in arid rangelands. Journal of Vegetation Science 10: 683-696.

Lavorel, S., S. McIntyre and K. Grigulis. 1999. Plant response to disturbance in a Mediterranean grassland: How many functional groups? Journal of Vegetation Science 10: 661-672.

Legris, P. and V.M. Meher-Homji. 1984. The Eastern Ghats: Phytogeogrphic aspects. Indian Review of Life Sciences 4:115-136.

Li, C., X. Zhang, X. Liu, O. Luukkanen and F. Berninger. 2006. Leaf morphological and physiological responses of Quercus aquifolioides along an altitudinal gradient. Silva Fennica 40 (1): 5-13.

Li, X. J. Li and P.S. Ashton. 1994. Dipterocarpaceae; p. 48-54 In W. Zhengyi, P.H. Raven and H. Deyuan (ed.). Flora of China, Vol 13. Beijing and Sr. Louis: Science Press / Missouri Botanical Garden.

Malhado, A.C.M., Y. Malhi, R.J. Whittaker, R.J. Ladle, H. ter Steege, O.L. Phillips, N. Butt, L.E.O.C. Aragão, C.A. Quesada, A. Araujo-Murakami, L. Arroyo, J. Peacock, G. Lopez-Gonzalez, T.R. Baker, L.O. Anderson, S. Almeida, N. Higuchi, T.J. Killeen, A. Monteagudo, D. Neill, N. Pitman, A. Prieto, R.P. Salomão, R. Váasquez-Martínez and W.F. Laurance. 2009. Spatial trends in leaf size of Amazonian rainforest trees. Biogeosciences 6: 1563-1576.

Matthew, K.M. 1983. The flora of the Tamil Nadu Carnatic. Tiruchirapalli: The Rapinat Herbarium. St. Joseph's College. 2154 p.

McLaren, J.R. 2006. Effects of plant functional groups on vegetation dynamics and ecosystem properties. Arctic 59 (4): 449-452.

McNeely J.A., K.R. Miller, W.V. Reid, R.A. Mittermeier. and T.B. Werner. 1990. Conserving the world's biological diversity. Gland: IUCN.

Meher-Homji, V.M. 2001. Bioclimatology and plant geography of peninsular India. Jodhpur: Scientific Publishers (India). 199 p.

Mishra, R.K., V.P. Upadhyay, S. Bal, P.K. Mohapatra and R.C. Mohanty. 2006. 
Phenology of species of moist deciduous forest sites of Similipal biosphere reserve. Lyonia 11 (1): 5-17.

Moorcroft, P.R., G.C. Hurtt, and S.W. Pacala. 2001. A method for scaling vegetation dynamics: The ecosystem demography model (ED). Ecological Monographs 71: 557-585.

Mukhopadhyay, D. and K. Basak. 2009. The Eastern Ghats belt - A polycyclic granulite terrain. Journal of Geological Society of India 73(4): 489-518.

Muthumperumal, C. and N. Parthasarathy. 2009. Angiosperms, climbing plants in tropical forests of southern Eastern Ghats, Tamil Nadu, India. Check List 5(1): 92-111.

Naeem, S. 2002. Ecosystem consequences of biodiversity loss: the evolution of a paradigm. Ecology 83: 1537-1552.

Naeem, S., F.S. Chair, Chapin III, R. Costanza, P.R. Ehrlich, F.B. Golley, D.U. Hooper, J.H. Lawton, R.V.O. Neill, H.A. Mooney, O.E. Sala, A.J. Symstad and D. Tilman. 1999. Biodiversity and ecosystem functioning: Maintaining natural life support processes. Issues in Ecology, 4: 1-12.

Nair, N.C. and A.N. Henry. (ed.). 1983. Flora of Tamil Nadu, India, Series I: Analysis, Vol. 1. Coimbatore: Botanical Survey of India. 188 p.

Natarajan, D., S.J. Britto, B. Balaguru, N. Nagamurugan and S. Soosairaj. 2004. Identification of conservation priority sites using remote sensing and GIS - A case study from Chitteri Hills, Eastern Ghats, Tamil Nadu. Current Science 86(9): 1316-1323.

Newton, P.N. 1988. The structure and phenology of a moist deciduous forest in the Central Indian Highlands. Vegetatio 75: 3-16.

Niinemets, U., A. Portsmuth, D. Tena, M. Tobias, S. Matesanz and F. Valladares. 2007. Do we underestimate the importance of leaf size in plant economics? Disproportional scaling of support costs within the spectrum of leaf physiognomy. Annals of Botany 100: 283-303.

NRSC. 2006. Land Use/Land Cover database on 1:50,000 scale, Natural Resources Census Project, LUCMD, LRUMG, RS and GIS AA, Hyderabad: National Remote Sensing Centre, ISRO.

Patel, A. 1997. Phenological patterns of Ficus in relation to other forest trees in southern India. Journal of Tropical Ecology 13 (5): 681-695.

Pragasan, L. A. and N. Parthasarathy. 2009. Angiosperms, tree species in tropical forests of southern Eastern Ghats, Tamil Nadu, India. Check List 5(3):542-569.

Rao D.M. and T. Pullaiah. 2007. Ethnobotanical studies on some rare and endemic floristic elements of Eastern Ghats-hill ranges of south east Asia, India. Ethnobotanical Leaflets International Web Journal 11: 5270.

Raunkiaer, C. 1934. The life forms of plants and statistical plant geography. Oxford: Clarendon Press. 632 p.

Reddy, C. S., S. Babar, A. Giriraj, K.N. Reddy and K.T. Rao. 2008. Structure and floristic composition of tree diversity in tropical dry deciduous forest of Eastern Ghats, Southern Andhra Pradesh, India. Asian Journal of Scientific Research 1: 57-64.

Reddy, C.S., K.N. Reddy, E.N. Murthy and V.S. Raju. 2009. Tree wealth of Eastern Ghats of Andhra Pradesh, India: An updated checklist. Check List 5(2):173-194.

Reich, P.B. and R. Borchert. 1982. Phenology and ecophysiology of the tropical tree, Tabebuia neochrysantha (Bignoniaceae). Ecology 63: 294-299.

Reich, P.B., M.B. Walters and D.S. Ellsworth. 1997. From tropics to tundra: Global convergence in plant function. Proceedings of the National Academy of Sciences 94: 13730-13734.

Richards, P.W. 1957. The tropical rainforest: An ecological study. Cambridge: Cambridge University Press. 450 p.

Roderick, M.L., S.L. Berry, A.R. Saunders and I.R. Noble. 1999. On the relationship between the composition, morphology and function of leaves. Functional Ecology 13: 697-710.

Rodgers, W.A. and H.S. Panwar. 1988. Planning a wildlife protected area network in India. 2 vols. Dehra Dun: Project FO: IND/82/003. FAO. $339.267 \mathrm{p}$.

Royer D.L., J.C. McElwain, J.M. Adams and P. Wilf. 2008. Sensitivity of leaf size and shape to climate within Acer rubrum and Quercus kelloggii. New Phytologist 179: 808-817.

Royer, D.L., P. Wilf, D.A. Janesko, E.A. Kowalski and D.L. Dilcher. 2005. Correlations of climate and plant ecology to leaf size and shape: potential proxies for the fossil record. American Journal of Botany 92(7): 1141-1151.

Saldanha, C.J. 1984. Flora of Karnataka. Volume 1. Magnoliaceae to Fabaceae. New Delhi: Oxford \& IBH. 535 p.

Singh, K.P. and C.P. Kushwaha. 2005. Paradox of leaf phenology: Shorea robusta is a semi-evergreen species in tropical dry deciduous forests in India. Current Science 88 (11): 1820-1824.
Soosairaj, S., S.J. Britto, B. Balaguru, N. Nagamurugan and D. Natarajan. 2004. Mapping forest types of Pacchaimalai Hills, Eastern Ghats, India using Remote sensing and GIS. Ecology, Environment and Conservation 10(2): 131-135.

Soosairaj, S., S.J. Britto, B. Balaguru, N. Nagamurugan, and D. Natarajan. 2007. Zonation of conservation priority sites for effective management of tropical forests in India: A value-based conservation approach. Applied Ecology and Environmental Research 5(2): 37-48.

Tanner E.V.J. 1980. Studies of the biomass and productivity in a series of montane rain forests in Jamaica. Journal of Ecology 68: 573-588.

Thomas, P.A. 2000. Trees: Their natural history. Cambridge: Cambridge University Press. 289 p.

Tilman, D., K. Johannes, W. David, P. Reich, R. Mark and S. Evan. 1997. The influence of functional diversity and composition on ecosystem processes. Science 277: 1300-1302.

Tiwari, D.N. 1995. A Monograph on Sal (Shorea robusta Geartn. f.). New Delhi: Vedams eBooks (P) Ltd. 277 p.

Turner, I. M. and H.T.M. Tan. 1991. Habitat-related variation in tree leaf form in four tropical forest types on Pulau Ubin, Singapore. Journal of Vegetation Science 2: 691-698.

Tutin, C.E.G. and M. Fernandez. 1993. Relationships between minimum temperature and fruit production in some tropical forest trees in Gabon. Journal of Tropical Ecology 9: 241-248.

Vaughan, R.E. and P.O. Wiehe. 1939. Studies on the vegetation of Mauritius: II. The effect of environment on certain features of leaf structure. The Journal of Ecology 27(2): 263-281.

Wackernagel, M., B. Schulz, D. Deumling, Callejas A. Linares, M. Jenkins, V. Kapos, C. Monfreda, J. Loh, N. Myers, R. Norgaard and J. Randers. 2002. Tracking the ecological overshoot of the human economy. Proceedings of the National Academy of Sciences 99(14): 9266-9271.

Webb, L.J. 1959. A physiognomic classification of Australian rain forests. Journal of Ecology 47:551-570.

Wilf, P., S.L. Wing, D.R. Greenwood and C.L. Greenwood. 1998. Using fossil leaves as paleoprecipitation indicators: An Eocene example. Geology 26 (3): 203-206.

Williams-Linera, G. 1997. Phenology of deciduous and broadleavedevergreen tree species in a Mexican tropical lower montane forest. Global Ecology and Biogeography Letters 6 (2): 115-127.

Wolfe, J.A. 1971. Tertiary climatic fluctuations and methods of analysis of tertiary floras. Palaeogeography, Palaeoclimatology, Palaeoecology 9: 27-57.

Wolfe, J.A. 1990. Palaeobotanical evidence for a major temperature increase following the Cretaceous/Tertiary boundary. Nature 343: 153-156.

Wolfe, J.A. 1995. Paleoclimatic estimates from Tertiary leaf assemblages. Annual Review of Earth and Planetary Sciences 23: 119-142.

Wolfe J.A. and G.R. Upchurch, jr. 1987a. Leaf assemblages across the Cretaceous-Tertiary boundary in the Raton Basin, New Mexico and Colorado. Proceedings of the National Academy of Sciences of the United States of America 84 (15): 5096-5100.

Wolfe J.A. and G.R. Upchurch, jr. 1987b. North American nonmarine climates and vegetation during the Late Cretaceous. Palaeogeography, Palaeoclimatology, Palaeoecology 61: 33-77.

Wright, I.tJ., P.B. Reich, J.H.C. Cornelissen, D.S. Falster, P.K. Groom, K. Hikosaka, W. Lee, C.H. Lusk, Ü. Niinemets, J. Oleksyn, N. Osada, H. Poorter, D.I. Warton and M. Westoby. 2005. Modulation of Leaf Economic Traits and Trait Relationships by Climate. Global Ecology and Biogeography 14: 411-421.

Wright, I.J., P.B. Reich, M. Westoby, D.D. Ackerly, Z. Baruch, F. Bongers, J. Cavender-Bares, F.S. Chapin, J.H.C. Cornelissen, M. Diemer, J. Flexas, E. Garnier, P.K. Groom, J. Gulias, K. Hikosaka, B.B. Lamont, T. Lee, W. Lee, C. Lusk, J.J. Midgley, M.-L. Navas, Ü. Niinemets, J. Oleksyn, N. Osada, H. Poorter, P. Poot, L. Prior, V.I. Pyankov, C. Roumet, S.C. Thomas, M.G. Tjoelker, E.J. Veneklaas. and R. Villar. 2004. The world-wide leaf economics spectrum. Nature 428: 821-827.

Wright, S.J. and C.P. van Schaik. 1994. Light and the phenology of tropical trees. American Naturalist 143: 192-199.

RECEIVED: January 2011

ACCEPTED: November 2012

PUBLISHED ONLINE: December 2012

EDITORIAL RESPONSIBILITY: Angelo Gilberto Manzatto

SUPPORTING INFORMATION. A complete dataset of the woody species from this study can be accessed at http://www.checklist.org.br/ getpdf?SL003-11A 







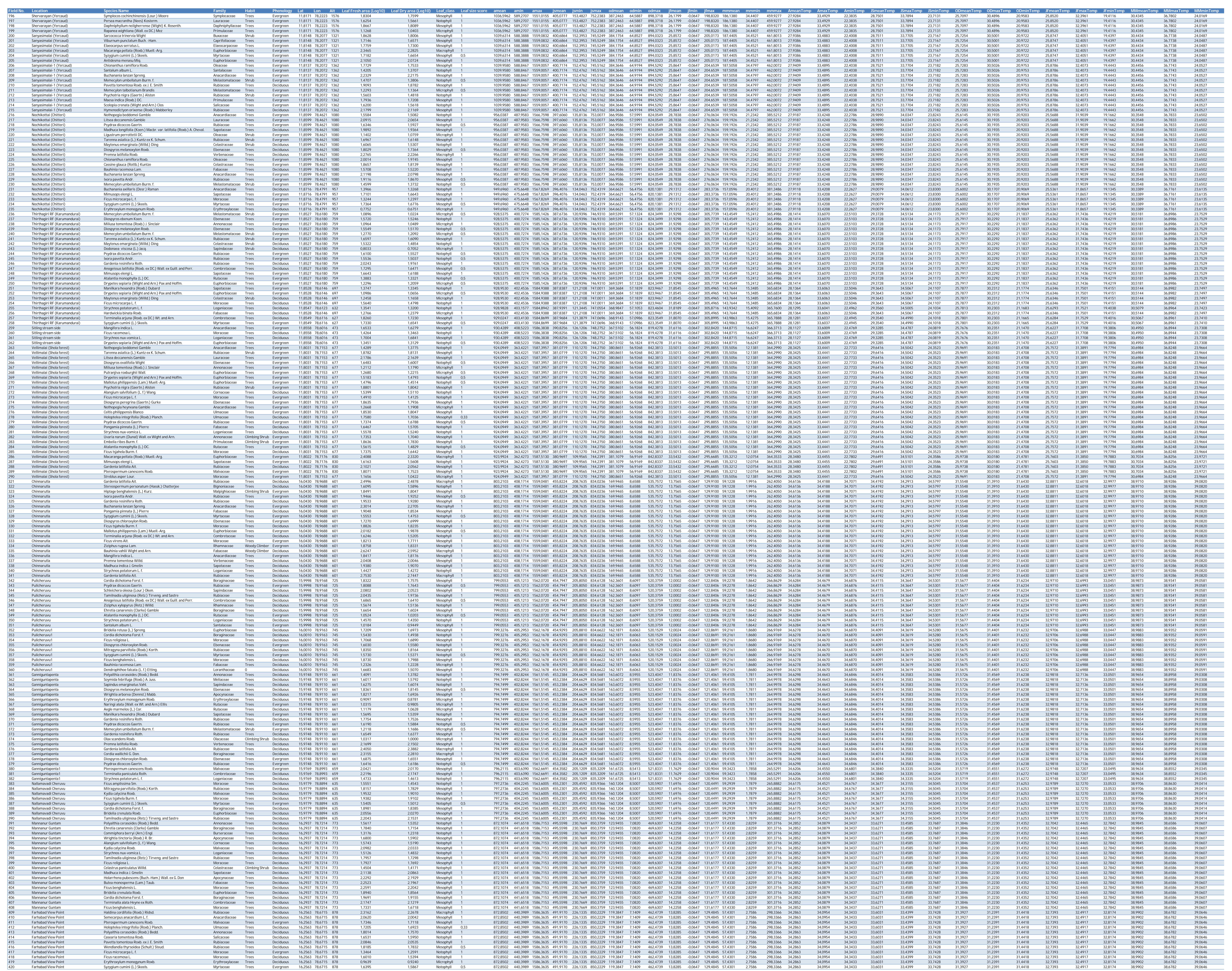

